\title{
Principles of Antibacterial Dosing in Continuous Renal Replacement Therapy
}

\author{
Gordon Choi $^{\mathrm{a}}$ Charles D. Gomersalla ${ }^{\mathrm{a}}$ Qi Tian ${ }^{\mathrm{a}}$ Gavin M. Joynt $^{\mathrm{a}}$ \\ Alexander M.M.Y. Li ${ }^{a} \quad$ Jeffrey Lipman ${ }^{b}$ \\ a Department of Anaesthesia and Intensive Care, The Chinese University of Hong Kong, Prince of Wales Hospital, \\ Shatin, Hong Kong SAR, China; ${ }^{b}$ Burns, Trauma and Critical Care Research Centre, University of Queensland, \\ Royal Brisbane and Women's Hospital, Herston, Qld., Australia
}

\section{Key Words}

Acute renal failure $\cdot$ Antibacterial agents $\cdot$ Continuous

renal replacement therapy • Pharmacodynamics •

Pharmacokinetics

\begin{abstract}
Background: Appropriate antibacterial therapy is important to maximize patient survival in sepsis. Acute renal failure complicates optimal antibiotic administration. Methods: MEDLINE search from 1986 to 2010 using the terms 'acute renal failure', 'pharmacokinetics', 'clearance', 'dosage', 'h(a)emofiltration', 'h(a)emodialysis', 'h(a)emodiafiltration', 'continuous renal replacement therapy', 'antibiotics', 'intensive care' and 'critically ill'. Results: Maximal bacterial killing and minimization of side effects depend on achieving pharmacokinetic targets appropriate to the selected antibacterial agent. Volume of distribution and clearance may be altered by critical illness and/or acute kidney injury. Clearance is determined by nonrenal clearance, residual renal clearance and continuous renal replacement therapy dose. Sieving and saturation coefficients are membrane specific, but may be altered by changes in protein binding induced by critical illness. A significant proportion of studies failed to report the essential dataset required for adequate antibacterial dosage calculation. Conclusions: Individualized dosing
\end{abstract}

based on first principles may be the most appropriate method of dosing, particularly when enhanced by therapeutic drug monitoring.

Copyright $\odot 2010$ S. Karger AG, Basel

Sepsis is the single most common cause for acute kidney injury (AKI) in the critically ill $[1,2]$. Despite limited prospective data, there is evidence to indicate that these patients have greater physiological disturbance, more severe organ dysfunction and higher mortality compared to those with nonseptic AKI $[2,3]$. As a result, optimal dosing of antibacterials in relation to the in vitro minimum inhibitory concentration (MIC) may be even more important to maximize bacterial killing while minimizing toxicity [4]. Unlike many other drugs, where the dose can be individually titrated to be effective, the optimal dose of antibacterials is currently based on theoretical considerations and data are largely derived from noncritically ill patients. To appropriately dose critically ill patients with AKI a thorough understanding of the relevant issues is required.

The major issues related to dosing of antibacterials in critically ill patients with AKI are that antibacterial pharmacokinetics may be altered by both acute renal failure and critical illness and are affected by renal replacement

\section{KARGER}

Fax +4161306 1234

E-Mail karger@karger.ch

www.karger.com
(C) 2010 S. Karger AG, Basel

0253-5068/10/0303-0195\$26.00/0

Accessible online at:

www.karger.com/bpu
Dr. Gordon Choi

Department of Anaesthesia and Intensive Care, Prince of Wales Hospital

The Chinese University of Hong Kong

Shatin, Hong Kong, SAR (China)

Tel. +852 2632 2734, Fax +852 2637 2422, E-Mail gchoi@ cuhk.edu.hk 
Table 1. Equations for calculating CRRT clearance from the first principles

\begin{tabular}{ll}
\hline Mode of CRRT & Calculation of CRRT clearance \\
\hline CVVH (postdilution) & $\mathrm{Cl}_{\mathrm{CVVH}}($ post $)=\mathrm{Q}_{\mathrm{f}} \times \mathrm{S}_{\mathrm{c}}$ \\
CVVH (predilution) & $\mathrm{Cl}_{\mathrm{CVVH}}($ pre $)=\mathrm{Q}_{\mathrm{f}} \times \mathrm{S}_{\mathrm{c}} \times \mathrm{Q}_{\mathrm{b}} /\left(\mathrm{Q}_{\mathrm{b}}+\mathrm{Q}_{\text {rep }}\right)$ \\
CVVHD & $\mathrm{Cl}_{\mathrm{CVVHD}}=\mathrm{Q}_{\mathrm{d}} \times \mathrm{S}_{\mathrm{d}}$ \\
$\mathrm{CVVHDF}$ & $\mathrm{Cl}_{\mathrm{CVVHDF}}=\left(\mathrm{Q}_{\mathrm{f}}+\mathrm{Q}_{\mathrm{d}}\right) \times \mathrm{S}_{\mathrm{d}}$ \\
\hline
\end{tabular}

$\mathrm{Cl}_{\mathrm{CVVH}}($ post $)=$ Clearance from CVVH using post-filter hemodilution; $\mathrm{Q}_{\mathrm{f}}=$ ultrafiltrate rate; $\mathrm{Cl}_{\mathrm{CVVH}}$ (pre) = clearance from CVVH using pre-filter hemodilution; $\mathrm{Q}_{\mathrm{b}}=$ blood flow rate; $\mathrm{Q}_{\text {rep }}=$ predilution replacement rate; $\mathrm{Cl}_{\mathrm{CVVHD}}=$ clearance from $\mathrm{CVVHD}$; $\mathrm{Cl}_{\mathrm{CVVHDF}}=$ clearance from CVVHDF; $\mathrm{Q}_{\mathrm{d}}=$ dialysate flow rate.

therapy. Renal replacement therapy in the critically ill is not a homogeneous entity, instead there is considerable variation in mode and dose and a discrepancy between prescribed and actual dose and the relationship between pharmacokinetics and pharmodynamics of individual antibacterials needs to be taken into account. We will review the relevant literature and propose a systematic approach to antibacterial dosing that takes these factors into account.

\section{Pharmacokinetic Factors}

Following administration of a drug, the initial fall in concentration is due to the distribution of the drug through the various body compartments. The extent of this distribution is reflected by the volume of distribution (Vd). Thereafter falls in concentration are predominantly dependent on clearance.

Sepsis is a complex process which is commonly initiated by endotoxins. It is characterized by the activation of multiple endogenous mediators, such as cytokines, interleukins, platelet-activating factor, eicosanoids, complement components and kinins $[5,6]$. Vascular endothelial dysfunction occurs either directly or indirectly, resulting in an increased capillary permeability. In conjunction with the maldistribution of blood flow, capillary leak syndrome ensues $[7,8]$. Leakage of fluid from the intravascular compartment to the interstitial space would be expected to increase $\mathrm{Vd}$ and reduce serum concentration of hydrophilic drugs such as $\beta$-lactams and aminoglycosides $[9,10]$. However, experimental data suggest the situation is more complex. For example, the Vd of ceftriaxone appears to be increased by critical illness and sep- sis [11]; however, the Vd of meropenem is not [12-18]. Amikacin Vd is higher in critically ill septic patients than in healthy volunteers [19].

AKI may also affect antibacterial pharmacokinetics. In addition to a reduction in renal clearance, it may result in changes in Vd. For example, ceftriaxone [11], ceftazidime $[20,21]$ and netilmicin [19] Vd are increased in patients with acute renal failure.

Disease states which are common amongst the critically ill, such as uremia, cirrhosis, nephrotic syndrome, epilepsy, hepatitis and severe burns, have been shown to decrease protein binding of drugs. In addition, systemic $\mathrm{pH}$, heparin, free fatty acids, and drugs such as salicylate and sulfonamide may act as competitive displacers for drug binding [22].

While Vd determines the initial or loading dose, subsequent doses are determined by total drug clearance. In patients with acute renal failure, total clearance is the sum of continuous renal replacement therapy (CRRT) and non-CRRT clearance (mainly residual renal and hepatic clearance).

CRRT is now typically performed via the venovenous route either as continuous venovenous hemofiltration $(\mathrm{CVVH})$, hemodialysis or a combination of the two - hemodiafiltration (CVVHDF). In CVVH, the predominant mechanism of solute removal is by means of convective clearance where hydrostatic pressure gradient is generated across a filter membrane. Replacement fluid can be added to the circuit either before blood reaches the membrane (predilution) or after passage over the filter membrane (postdilution). For CVVH operating in postdilution mode, a correction factor (CF) needs to be used to calculate clearance $\left(C F=Q_{b} / Q_{b}+Q_{\text {rep }}\right)$ where $Q_{b}$ and $\mathrm{Q}_{\text {rep }}$ represent blood flow rate and replacement rate, respectively.

In contrast, the predominant mechanism of solute removal in continuous venovenous hemodialysis is by means of diffusive clearance. This is achieved by generating a continuous concentration gradient using a countercurrent flow of plasma and dialysate fluid, between which equilibration occurs. In CVVHDF, the two mechanisms of convective and diffusive clearance are combined to provide solute removal. The major factors determining clearance by each mode of CRRT are given in table 1 .

The concentration of drug in ultrafiltrate or dialysate relative to plasma is expressed as sieving coefficient $\left(\mathrm{S}_{\mathrm{c}}\right)$ or saturation coefficient $\left(\mathrm{S}_{\mathrm{d}}\right)$, respectively. Passage of antibacterials across the filter membrane is essentially independent of drug molecular weight as the pore size of modern membranes vastly exceeds the size of most com- 
monly used antibacterials. As a result, drug protein binding is the major determinant of both $S_{c}$ and $S_{d}$. In general, $S_{c}$ ranges from 0 to 1 . It has been suggested that $S_{c}$ can be estimated from published values of protein binding $(\mathrm{PB})$, such that $\mathrm{S}_{\mathrm{c}}=1-\mathrm{PB}$. Measured $\mathrm{S}_{\mathrm{c}}$ and $\mathrm{S}_{\mathrm{c}}$ estimated from published values of protein binding are correlated [23]. However, as discussed below, protein binding in the critically ill is variable and for some drugs (e.g. levofloxacin) $S_{c}$ varies widely [24-28]. Furthermore, $S_{c}$ may be affected by membrane material, drug-membrane interactions and flux properties. Similar to $S_{c}$, the value of $S_{d}$ for any given drug may be membrane specific and subject to drug-membrane interactions. An increase in unbound drug will increase $S_{c}$ and $S_{d}$ and hence elimination by CRRT. For example, the unbound fraction of ceftriaxone is increased in patients with critical illness and is further increased by renal failure [11]. As a result, clearance of ceftriaxone by CRRT is likely to be higher than would be expected from protein binding in healthy volunteers, and this is confirmed by experimental data [29, 30]. Furthermore, $S_{d}$ may be affected by blood and dialysate flow. At high blood flow the transit time may be insufficient for complete equilibration across the membrane, particularly for filters with a small surface area, resulting in a decrease in $S_{d}$ [31]. Similarly, an increase in dialysate flow rate will reduce drug concentration in the dialysate and hence decrease $S_{d}$. Nevertheless, at the blood (150-200 $\mathrm{ml} / \mathrm{min}$ ) and dialysate flow rates $(<35 \mathrm{ml} / \mathrm{min})$ typically used during CRRT, clearance is linearly related to dialysate flow rate indicating that $S_{d}$ is constant under these conditions [32].

In general, drugs with a large $\mathrm{Vd}$ are poorly eliminated by CRRT as the plasma concentration of drug is low relative to the amount of drug in the body. This has led to the recommendation that supplemental dosing of these drugs is unnecessary [33]. However, for the lipophilic fluoroquinolones, such as ciprofloxacin and levofloxacin, Vds are large ( $>1.5$ liters $/ \mathrm{kg}$ ) but renal clearance still accounts for $\geq 70 \%$ of total clearance $[34,35]$. The elimination half-life of both drugs approaches that of normal healthy volunteers with increasing ultrafiltration and/or dialysate flow rate necessitating higher daily doses than previously recommended [25]. If, however, the reason for increased $\mathrm{Vd}$ is a fall in protein binding, elimination by CRRT (and kidneys) will be affected by an increase in the free fraction of the drug. In general, drugs with a high $\mathrm{Vd}$ $(>1$ liter $/ \mathrm{kg})$ and high protein binding $(>80 \%)$ are poorly eliminated by CRRT [36].

The Gibbs-Donnan effect refers to the effect of retained anionic protein (such as albumin) on the blood side of the filter membrane. This could lead to transient retention of cationic drugs such as aminoglycosides and levofloxacin. Anionic drugs such as ceftazidime and cefotaxime may be repelled [22]. However, the clinical relevance of this effect is unclear $[23,37,38]$. Adsorption of antibacterials to the filter may also result in drug elimination. Limited in vitro data suggest that adsorption is both membrane and drug dependent [37-39]. At clinically relevant concentrations, adsorption of levofloxacin and vancomycin is unlikely to be clinically significant $[37,39]$; however, a significant amount of amikacin binds irreversibly to sulfonated polyacrylonitrile membranes in vitro [38]. The clinical importance of adsorption of antibacterials is currently unknown but warrants further investigation.

Non-CRRT clearance is predominantly determined by residual renal function and hepatic clearance. However, alternative elimination pathways such as transintestinal excretion (e.g. for ciprofloxacin) may represent compensatory mechanisms that prevent accumulation in patients with renal failure [40]. Renal elimination is to a large extent determined by glomerular filtration rate, with contributions from tubular secretion and reabsorption, but the ability to upregulate clearance is relatively limited. Hepatic clearance, however, may be variable depending on the degree of underlying organ failure. Hepatic dysfunction not only occurs in those with acute liver failure or decompensated chronic liver disease, but it is also a common entity in the critically ill $[41,42]$. In contrast, for those without concomitant hepatic dysfunction, hepatic drug clearance may actually increase in the presence of acute renal failure [43, 44]. Quantitative or at least qualitative evaluation of hepatic function should therefore be considered prior to formulating an antibacterial dosing regime, in particular for agents with multiple routes of clearance (i.e. ciprofloxacin and meropenem).

The relationship between pharmacokinetic endpoints and pharmacodynamic outcomes varies between classes of antibacterials. Understanding of these relationships is important in determining dosing regimes in order to maximize efficacy, minimize the development of resistance and minimize the risk of toxicity [10]. Broadly, antibacterials fall into three groups: those with time-dependent killing, those with concentration-dependent killing and those with both time- and concentration-dependent killing. These kill characteristics have been previously determined under in vitro conditions. For each group, there is a pharmacokinetic target that is most closely associated with optimal killing. These targets are often quoted in reference to the MIC (table 2) and can be used to guide dosing regimes [45]. 
Table 2. Kill characteristics of different antibacterials and pharmacokinetic targets associated with optimal bacterial killing

\begin{tabular}{|c|c|c|}
\hline Antibacterials & Killing characteristics & Pharmacokinetic targets \\
\hline$\beta$-Lactams & time dependent & $40-100 \%$ of dosing interval $>$ MIC or $40-100 \%$ of dosing interval $>5$ times MIC [47] \\
\hline Aminoglycosides & concentration dependent & $\mathrm{C}_{\text {max }}: \mathrm{MIC} 8-10[53]$ \\
\hline Fluoroquinolones & $\begin{array}{l}\text { concentration dependent/ } \\
\text { time dependent }\end{array}$ & $\mathrm{C}_{\text {max }}: \mathrm{MIC} 6-8, \mathrm{AUC}_{24}: \mathrm{MIC} 100-125$ (Gram-negatives), 34 (S. pneumoniae) $[87,91]$ \\
\hline Vancomycin & concentration dependent & $\mathrm{AUC}_{24}: \mathrm{MIC} \geq 400$ (vs. Staphylococcus aureus) [77] \\
\hline Linezolid & concentration dependent & $\mathrm{AUC}_{24}: \mathrm{MIC} 50$ (S. pneumoniae), $\mathrm{AUC}_{24}: \mathrm{MIC} 82$ (S. aureus) [92] \\
\hline $\begin{array}{l}\text { Macrolides, azalides, } \\
\text { ketolides }\end{array}$ & concentration dependent & $\begin{array}{l}\text { probably } \mathrm{AUC}_{24}: \mathrm{MIC} \text { (drug concentration at target site). Relevance of plasma } \\
\text { concentrations doubtful given the fact that drugs are concentrated in tissue [90] }\end{array}$ \\
\hline Metronidazole & concentration dependent & not established \\
\hline
\end{tabular}

The $\beta$-lactams, comprising penicillins, cephalosporins, carbapenems and monobactams, are the classic example of antimicrobials which exhibit time-dependent killing. They have a slow continuous bactericidal effect and killing is most closely related to the duration of time for which the serum concentration exceeds a threshold concentration. Further increases in concentration above the threshold do not increase killing. The appropriate threshold is not clearly established but varies between 1 and 5 times MIC [46]. The duration of time for which the serum concentration should exceed the threshold is also unclear with recommendations ranging from 40 to $100 \%$ of the dosing interval [47] depending on the target organism and the choice of $\beta$-lactams. The optimal duration is, in part, related to the postantibiotic effect of $\beta$-lactams. Postantibiotic effect refers to the continued suppression of bacterial growth even when drug concentrations fall below the MIC of the bacteria [48]. $\beta$-Lactams have a significant postantibiotic effect against Gram-positive organisms but, with the exception of carbapenems, not Gram-negatives. As a result, it has been proposed that, in the absence of any postantibiotic effect, the serum concentration of $\beta$-lactam antibacterial agent should exceed the MIC for the respective organism for $90-100 \%$ of the dosing interval [49]. However, for carbapenems, $40 \%$ may be sufficient [50]. Furthermore, resistant strains are likely to emerge when the antibacterial level falls below the MIC for more than half of the dosing interval [51].

Aminoglycosides are the classic example of concentration-dependent killing. Bactericidal activity is associated with the postdistribution peak plasma concentration $\left(\mathrm{C}_{\max }\right)$ to MIC ratio $\left(\mathrm{C}_{\max } / \mathrm{MIC}\right)$ both in vitro [52] and in vivo [53]. An optimal $\mathrm{C}_{\max } / \mathrm{MIC}$ ratio of $8-10$ has been recommended $[48,53-55]$, and this is best achieved by the extended interval dosing regime where larger doses are given less frequently. In patients with normal renal function, doses are often given once daily. Aminoglycosides demonstrate a significant postantibiotic effect $(>3 \mathrm{~h}$ ), the duration of which is concentration dependent $[53,56-61]$ and, therefore, the postantibiotic effect is enhanced by the administration of larger doses at extended time intervals. Furthermore, an extended interval dosing regime should result in lower trough concentrations and therefore reduce toxicity. This has been confirmed in clinical studies which have demonstrated a comparable, if not superior outcome with less toxicity compared to conventional multiple dosing regimes [62-65].

The fluoroquinolones (ciprofloxacin, levofloxacin and moxifloxicin) typically exhibit combined concentrationand time-dependent kill characteristics. Previous research has suggested that achieving a $\mathrm{C}_{\max } / \mathrm{MIC}$ ratio of 10 for fluoroquinolones is the critical variable in predicting bacterial eradication [66]. Others have proposed that dosing should maximize the ratio of area under the concentration time curve (AUC) to MIC in $24 \mathrm{~h}\left(\mathrm{AUC}_{24} /\right.$ MIC) [67]. An $\mathrm{AUC}_{24} / \mathrm{MIC}$ ratio of $>125$ and $>30$ has been recommended for Gram-negative and Gram-positive organisms, respectively [4]. Others have also shown that a high $\mathrm{AUC}_{24} / \mathrm{MIC}$ can reduce the development of resistance [68-70] and for Gram-negative organisms and that resistance may occur when the $\mathrm{AUC}_{24} / \mathrm{MIC}$ is $<100$ $[23,71]$. Therefore, it is important that threshold $\mathrm{AUC}_{24} /$ MIC ratios need to be obtained for the optimal dosing of fluroquinolones. 
Avoidance of toxicity is an important consideration when designing dose regimens. However, while toxicity is associated with drug concentrations, the relationship is not simple and is dependent on a large number of factors, which may vary from agent to agent [72-76]. High trough concentrations appear to be associated with aminoglycoside toxicity. Vancomycin toxicity has similarly been linked to trough concentrations; however, these data are based on only 8 patients with evidence of nephrotoxicity [77]. $\beta$-Lactam neurotoxicity is most closely associated with renal failure. This is in part due to decreased clearance but also increased CNS penetration as a result of altered protein binding and increased permeability of the blood-brain barrier [76, 78]. As a result, the relationship between blood concentration and toxicity may be complex. For example, cefepime neurotoxicity has been associated with a wide range of blood concentrations [79] and has even been reported in a patient with normal renal function and receiving a usual dose of cefepime [80]. Furthermore, there are no data relating specific serum pharmacokinetic endpoints with $\beta$-lactam toxicity. In rats, fluoroquinolone-induced seizures are associated with high peak plasma concentrations [81] and this militates against an extended-interval dosing strategy for fluoroquinolones aimed at maximizing $\mathrm{C}_{\max } / \mathrm{MIC}$ ratio instead of $\mathrm{AUC}_{24} / \mathrm{MIC}$ ratio.

\section{Critique of Currently Available Dosage Regimes}

Dosage recommendations for patients with renal dysfunction are available in most drug inserts, textbooks and antibiotic guidelines. Pharmacokinetic data are either derived from healthy individuals or those with chronic renal failure with dosage adjustment based on creatinine clearance. Estimated glomerular filtration rate may be used as a surrogate for creatinine clearance and the average daily effluent rate can be substituted for the glomerular filtration rate. However, it is important to note that recommendations for patients with chronic renal failure undergoing intermittent renal replacement therapy are not appropriate for the critically ill because of differences in the mode of renal replacement therapy as well as pharmacokinetic differences [82]. Other recommendations use a fixed and suboptimal effluent rate [83] or do not take the dose of CRRT into account [33].

An alternative approach is to use doses recommended in studies of the pharmacokinetics of antibacterials in critically ill patients receiving CRRT. However, dosages based on the findings of individual studies may not be appropriate unless the mode and dose of CRRT being used are similar to those in the study. The mode and dose of CRRT are highly variable, not only between studies but also within studies (table 3 ). In a recent review of the literature, $12 \%$ of studies reviewed omitted even basic information such as ultrafiltrate/dialysate rate and $14 \%$ made no dose recommendation. Furthermore, in only $8 \%$ was the dose recommendation referenced to a pharmacokinetic target based on MIC [84].

There is also a number of dose adjustment equations proposed for critically ill patients requiring CRRT (table 4). These equations either assume that measurement of antibacterial concentrations is widely available or that $\mathrm{S}_{\mathrm{c}}$ can be accurately estimated from data on protein binding obtained from the noncritically ill or are based on either downward adjustment from those with normal renal function or upward adjustment from those with chronic renal failure with anuria. However, 'normal' doses or doses recommended for anuric patients may not achieve pharmacokinetic targets associated with improved outcome. For example, based on pharmacokinetic data in critically ill patients [85] and the MIC for Streptococcus pneumoniae [86], the recommended dose of moxifloxacin may not achieve an optimal AUC:MIC $>33.7$ [87] in geographical localities in which the MIC is high but still below accepted breakpoints. Limitations of these formulae and their inherent flaws have been previously highlighted [88], with method 4 (table 4) consistently overestimating daily dosing needs for drugs with low nonrenal clearance and $>3$-fold difference in the calculated dose of gentamicin and vancomycin between methods 3 and 4 (table 4).

From the above discussion, it can be seen that there are many factors that need to be taken into account when determining appropriate doses of antibacterial agents for critically ill patients receiving CRRT. None of the aforementioned dosing recommendations take all these factors into account. Given the variability in mode, dose of CRRT and the MICs in different ICUs, we believe the most appropriate way to dose antibacterials in patients receiving CRRT is to calculate an appropriate individualized dose from first principles (fig. 1).

As previously stated the initial dose is dependent on the $\mathrm{Vd}$ and maintenance doses are dependent on total clearance (CRRT and non-CRRT clearance). Furthermore, antibacterial dosing needs to be adjusted according to the pharmacokinetic target associated with optimal killing. Thus appropriate dose calculation requires knowledge of the pharmacokinetic target (table 2) and the usual MIC of the suspected organism at the prescrib- 
Table 3. Pharmacokinetic data for antibacterials commonly used in intensive care in patients receiving CRRT

\begin{tabular}{|c|c|c|c|c|c|}
\hline Drug (ref. No.) & $\begin{array}{l}\text { Mode of CRRT } \\
\text { (number of } \\
\text { patients) }\end{array}$ & Residual renal function & $\mathrm{Vd}$ & Non-CRRT clearance ${ }^{1}$ & $\begin{array}{l}\text { Membrane/ } \\
\text { surface area }\end{array}$ \\
\hline \multicolumn{6}{|l|}{$\begin{array}{l}\text { Penicillins } \\
\text { Ampicillin (no data) }\end{array}$} \\
\hline Amoxicillin [93] & CVVH (12) & $\mathrm{U} / \mathrm{O}<400 \mathrm{ml} / 12 \mathrm{~h}$ & NS & NS & $\mathrm{CT} / 1.9 \mathrm{~m}^{2}$ \\
\hline Piperacillin [94] & CVVH (6) & $\begin{array}{l}4 \text { anuric patients } \\
2 \text { patients } \mathrm{U} / \mathrm{O}<400 \mathrm{ml} / 24 \mathrm{~h}\end{array}$ & $0.48 \pm 0.24$ liter $/ \mathrm{kg}$ & NS & $\mathrm{PS} / 0.5 \mathrm{~m}^{2}$ \\
\hline Piperacillin [94] & CVVH (4) & $\begin{array}{l}3 \text { anuric patients } \\
1 \text { patient } \mathrm{U} / \mathrm{O}=220 \mathrm{ml} / 24 \mathrm{~h}\end{array}$ & $0.14 \pm 0.07$ liter $/ \mathrm{kg}$ & NS & $\mathrm{PS} / 0.5 \mathrm{~m}^{2}$ \\
\hline Piperacillin [95] & CAVHD (12) & $\mathrm{Cl}_{\text {urea }} ; 18.4 \pm 2.3 \mathrm{ml} / \mathrm{min}$ & $25.8 \pm 3.8$ liters & $34.9 \pm 21.2$ & AN69/0.43 $\mathrm{m}^{2}$ \\
\hline $\begin{array}{l}\text { Piperacillin- } \\
\text { tazobactam [68] }\end{array}$ & CVVH (4) & $\mathrm{Cl}_{\mathrm{cr}}: 8.67 \pm 2.31 \mathrm{ml} / \mathrm{min}$ & $\begin{array}{l}\text { piperacillin: } 21.0 \pm 11.7 \text { liters, } \\
\text { tazobactam: } 18.9 \pm 7.1 \text { liters }\end{array}$ & $\begin{array}{l}\text { piperacillin: } 38.55 \\
\text { tazobactam: } 29.5\end{array}$ & AN69/0.9 $\mathrm{m}^{2}$ \\
\hline $\begin{array}{l}\text { Piperacillin- } \\
\text { tazobactam [68] }\end{array}$ & CVVH (5) & $\mathrm{Cl}_{\mathrm{cr}}: 25.20 \pm 7.73 \mathrm{ml} / \mathrm{min}$ & $\begin{array}{l}\text { piperacillin: } 26.8 \pm 19.8 \text { liters, } \\
\text { tazobactam: } 21.6 \pm 3.0 \text { liters }\end{array}$ & $\begin{array}{l}\text { piperacillin: } 78.4 \\
\text { tazobactam: } 46.3\end{array}$ & AN69/0.9 $\mathrm{m}^{2}$ \\
\hline $\begin{array}{l}\text { Piperacillin- } \\
\text { tazobactam [96] }\end{array}$ & CVVHD (8) & anuric & $\begin{array}{l}\text { piperacillin: } 0.31 \pm 0.07 \text { liter } / \mathrm{kg} \\
\text { tazobactam: } 0.24 \pm 0.09 \mathrm{liter} / \mathrm{kg}\end{array}$ & $\begin{array}{l}\text { piperacillin: } 49.5 \pm 60.1 \\
\text { tazobactam: } 23.7 \pm 14.9\end{array}$ & AN69/0.6 m ${ }^{2}$ \\
\hline $\begin{array}{l}\text { Ticarcillin- } \\
\text { clavulanate [97] }\end{array}$ & CVVH (3) & $\begin{array}{l}2 \text { patients anuric } \\
1 \text { patient } U / O>2 \text { liters/day }\end{array}$ & $\begin{array}{l}\text { ticarcillin: } 0.26 \text { liter } / \mathrm{kg}(\mathrm{n}=2 \text {, with ECMO }) \\
0.25 \text { liter } / \mathrm{kg}(\mathrm{n}=1 \text {, without ECMO) } \\
\text { clavulanate: } 0.86 \text { liter } / \mathrm{kg}(\mathrm{n}=2 \text {, with } \\
\text { ECMO), } 0.33 \text { liter } / \mathrm{kg}(\mathrm{n}=1 \text {, without ECMO })\end{array}$ & $\begin{array}{l}\text { ticarcillin: } 5.75(\mathrm{n}=2 \text {, with ECMO), } \\
40.83(\mathrm{n}=1 \text {, without ECMO) } \\
\text { clavulanate: } 61.75(\mathrm{n}=2 \text {, with ECMO), } \\
186.8(\mathrm{n}=1 \text {, without ECMO })\end{array}$ & $\mathrm{PS} / 0.25 \mathrm{~m}^{2}$ \\
\hline Flucloxacillin [93] & CVVH (5) & $\mathrm{U} / \mathrm{O}<400 \mathrm{ml} / 12 \mathrm{~h}$ & NS & NS & $\mathrm{CT} / 1.9 \mathrm{~m}^{2}$ \\
\hline Flucloxacillin [98] & CVVH (10) & anuric & $0.54 \pm 0.43$ liter $/ \mathrm{kg}$ & 106.9 & $\mathrm{PA} / 0.7 \mathrm{~m}^{2}$ \\
\hline \multicolumn{6}{|l|}{ Oxacillin (no data) } \\
\hline \multicolumn{6}{|l|}{ Nafcillin (no data) } \\
\hline \multicolumn{6}{|l|}{ Cloxacillin (no data) } \\
\hline \multicolumn{6}{|l|}{ Cephalosporins } \\
\hline Cefuroxime [99] & CAVHD (12) & NS & $22.8 \pm 9.2$ liters & 1.5 & AN69/0.43 $\mathrm{m}^{2}$ \\
\hline Cefuroxime [100] & CAVH (3) & $\mathrm{Cl}_{\mathrm{cr}}<1 \mathrm{ml} / \mathrm{min}$ & $21 \pm 2.5$ liters & $21 \pm 6.6$ & $\mathrm{PS} / 0.6 \mathrm{~m}^{2}$ \\
\hline \multicolumn{6}{|l|}{ Cefotaxime (no data) } \\
\hline Ceftazidime [99] & CAVHD (9) & NS & $31.1 \pm 14.6$ liters $(5)$ & 15.15 & AN69/0.43 $\mathrm{m}^{2}$ \\
\hline Ceftazidime [101] & CVVH (12) & anuric & $0.41 \pm 0.16$ liter $/ \mathrm{kg}$ & $66.57 \pm 12.90$ & $\mathrm{PS} / 0.7 \mathrm{~m}^{2}$ \\
\hline Ceftazidime [102] & $\begin{array}{l}\text { CVVH/CVVHD } \\
(8)\end{array}$ & $\mathrm{Cl}_{\mathrm{cr}}<20 \mathrm{ml} / \mathrm{min}$ & NS & NS & $\begin{array}{l}\text { AN69/0.6 m² } \\
\text { PMMA/2.1 m² } \\
\text { PS/0.65 m }\end{array}$ \\
\hline Ceftazidime [103] & CVVHDF (7) & $\mathrm{Cl}_{\mathrm{cr}} \leq 5 \mathrm{ml} / \mathrm{min}$ & $0.25 \pm 0.09 \mathrm{liter} / \mathrm{kg}$ & $28.9 \pm 5.6$ & AN69/0.6 m² \\
\hline Ceftazidime [21] & CVVHDF (2) & $\mathrm{Cl}_{\mathrm{cr}}=0 \mathrm{ml} / \mathrm{min}$ & 0.46 liter $/ \mathrm{kg}$ & 24.95 & AN69/0.9 m ${ }^{2}$ \\
\hline Ceftazidime [93] & CVVH (7) & $\mathrm{U} / \mathrm{O}<400 \mathrm{ml} / 12 \mathrm{~h}$ & NS & NS & $\mathrm{CT} / 1.9 \mathrm{~m}^{2}$ \\
\hline Ceftriaxone [104] & CVVH (6) & $\mathrm{Clcr}<10 \mathrm{ml} / \mathrm{min}$ & $0.42 \pm 0.21$ liter $/ \mathrm{kg}$ & $22.7 \pm 19.1$ & $\mathrm{PA} / 1.4 \mathrm{~m}^{2}$ \\
\hline Ceftriaxone [105] & CVVH (5) & ESRD & NS & NS & AN69/0.6 $\mathrm{m}^{2}$ \\
\hline Ceftriaxone [105] & CVVH (5) & ESRD & NS & NS & PMMA/2.1 $\mathrm{m}^{2}$ \\
\hline Ceftriaxone [105] & CVVH (5) & ESRD & NS & NS & $\mathrm{PS} / 0.65 \mathrm{~m}^{2}$ \\
\hline Cefepime [93] & CVVH (2) & $\mathrm{Cl}_{\mathrm{cr}}=29 \mathrm{ml} / \mathrm{min}(1)$ & 0.65 liter $/ \mathrm{kg}$ & NI & AN69/0.9 $\mathrm{m}^{2}$ \\
\hline Cefepime [106] & CVVHDF (2) & $\begin{array}{l}\mathrm{Cl}_{\mathrm{cr}}<10 \mathrm{ml} / \mathrm{min} \\
\mathrm{Cl}_{\mathrm{cr}}=35 \mathrm{ml} / \mathrm{min}\end{array}$ & 0.6 liter $/ \mathrm{kg}$ & NI & $\begin{array}{l}\text { AN69/0.9 } \mathrm{m}^{2} \\
\mathrm{PS} / 1.4 \mathrm{~m}^{2}\end{array}$ \\
\hline Cefepime [107] & CVVH (5) & $\mathrm{U} / \mathrm{O}<155 \mathrm{ml} / 24 \mathrm{~h}$ & $0.46 \pm 0.14$ liter $/ \mathrm{kg}$ & 23 & $\mathrm{AN} 69 / 0.6 \mathrm{~m}^{2}$ \\
\hline Cefepime [107] & CVVHDF (7) & $\mathrm{U} / \mathrm{O}<67 \mathrm{ml} / 24 \mathrm{~h}$ & $0.34 \pm 0.1$ liter $/ \mathrm{kg}$ & 21 & AN69/0.6 m² \\
\hline \multicolumn{6}{|l|}{ Cefoperazone (no data) } \\
\hline \multicolumn{6}{|l|}{$\begin{array}{l}\text { Monolactams } \\
\text { Aztreonam (no data) }\end{array}$} \\
\hline $\begin{array}{l}\text { Carbapenems } \\
\text { Imipenem [108] }\end{array}$ & CVVH (6) & $\begin{array}{l}2 \text { anuric patients } \\
4 \text { patients } \mathrm{U} / \mathrm{O}<43 \mathrm{ml} / 24 \mathrm{~h}\end{array}$ & $0.36 \pm 0.10$ liter $/ \mathrm{kg}$ & $109 \pm 24$ & AN69/0.6 $\mathrm{m}^{2}$ \\
\hline Imipenem [108] & CVVHDF (6) & $\begin{array}{l}2 \text { anuric patients } \\
4 \text { patients } \mathrm{U} / \mathrm{O}<135 \mathrm{ml} / 24 \mathrm{~h}\end{array}$ & $0.37 \pm 0.13$ liter $/ \mathrm{kg}$ & $120 \pm 32$ & AN69/0.6 $\mathrm{m}^{2}$ \\
\hline Imipenem-cilastatin [109] & CAVH (6) & $\begin{array}{l}2 \text { anuric patients } \\
4 \text { patients } \mathrm{U} / \mathrm{O}<350 \mathrm{ml} / 24 \mathrm{~h}\end{array}$ & $\begin{array}{l}\text { imipenem: } 0.29 \pm 0.03 \text { liter } / \mathrm{kg} \\
\text { cilastatin: } 0.27 \pm 0.07 \text { liter } / \mathrm{kg}\end{array}$ & $\begin{array}{l}\text { imipenem: } 108.5 \pm 29.6 \\
\text { cilastatin: } 20.6 \pm 30.3\end{array}$ & PS/NS \\
\hline Imipenem-cilastatin [110] & CVVH (12) & $\begin{array}{l}10 \text { anuric patients } \\
2 \text { patients } \mathrm{U} / \mathrm{O}=200 \mathrm{ml} / 8 \mathrm{~h}\end{array}$ & $\begin{array}{l}\text { imipenem: } 24.3 \pm 7.7 \text { liters } \\
\text { cilastatin: } 19.6 \pm 7.3 \text { liters }\end{array}$ & $\begin{array}{l}\text { imipenem: } 90.8 \pm 26.3 \\
\text { cilastatin: } 13.2 \pm 13.9\end{array}$ & AN69/NS \\
\hline
\end{tabular}




\begin{tabular}{|c|c|c|c|c|c|}
\hline \multicolumn{2}{|l|}{$\mathrm{S}_{\mathrm{c}}$} & \multirow[t]{2}{*}{$\mathrm{S}_{\mathrm{d}}$} & \multirow[t]{2}{*}{ Dose recommended by authors } & \multirow{2}{*}{$\begin{array}{l}\text { Total effluent rate (range, } \\
\text { unless otherwise specified) }\end{array}$} & \multirow[t]{2}{*}{ Remarks } \\
\hline pre & post & & & & \\
\hline $0.71 \pm 0.16$; route of dilution & NS & NA & NS & mean \pm SD: $29 \pm 7 \mathrm{ml} / \mathrm{kg} / \mathrm{h}$ & \\
\hline NS & NS & NS & 4 g 12 hourly & $0.76 \pm 0.20$ to $0.88 \pm 0.18$ liters $/ \mathrm{h}$ & $\begin{array}{l}\text { first-dose pharmaco- } \\
\text { kinetics }\end{array}$ \\
\hline NS & NS & NS & $4 \mathrm{~g} 12$ hourly & $0.50 \pm 0.08$ to $0.72 \pm 0.14$ liters $/ \mathrm{h}$ & \\
\hline NA & NA & $0.71 \pm 0.21$ & $150 \%$ of dose for anuric patients & mean \pm SD: $1.22 \pm 0.09$ liters $/ \mathrm{h}$ & \\
\hline $\begin{array}{l}\text { piperacillin: } 0.42 \pm 0.25 \\
\text { tazobactam: } 0.76 \pm 0.26\end{array}$ & NA & NA & NS & mean \pm SD: $1.63 \pm 0.47$ liters $/ h$ & \\
\hline $\begin{array}{l}\text { piperacillin: } 0.38 \pm 0.37 \\
\text { tazobactam: } 0.73 \pm 0.32\end{array}$ & NA & NA & NS & mean $\pm S D: 1.82 \pm 0.26$ liters $/ \mathrm{h}$ & \\
\hline NA & NA & $\begin{array}{l}\text { piperacillin: } 0.87 \pm 0.21 \\
\text { tazobactam: } 0.64 \pm 0.19\end{array}$ & NS & $1.58-1.70$ liters $/ \mathrm{h}$ & \\
\hline $\begin{array}{l}\text { ticarcillin: } 0.72(\mathrm{n}=2 \text {, with } \\
\text { ECMO), } 1.06(\mathrm{n}=1 \text {, without ECMO }) \\
\text { clavulanate: } 1.81(\mathrm{n}=2 \text {, with ECOM }) \text {, } \\
1.44(\mathrm{n}=1 \text {, without ECMO); route of } \\
\text { dilution NS }\end{array}$ & NS & & NS & $0.82-0.95$ liter $/ \mathrm{h}$ & $\begin{array}{l}\text { children (2 patients } \\
\text { receiving concomitant } \\
\text { ECMO, 1 patient } \\
\text { without ECMO U/O } \\
>2 \text { liters/day) }\end{array}$ \\
\hline $\begin{array}{l}0.33 \pm 0.34(0.2 \text { in } 4 \text { patients } \\
\text { and } 0.94 \text { in } 1 \text { patient }) \\
\text { route of dilution }\end{array}$ & NS & NA & NS & mean $\pm S D: 23 \pm 7 \mathrm{ml} / \mathrm{kg} / \mathrm{h}$ & \\
\hline NA & $0.21 \pm 0.09$ & NA & $4 \mathrm{~g} 8$ hourly & mean \pm SD: $3.42 \pm 0.54$ liters $/ \mathrm{h}$ & \\
\hline NA & NA & $0.90 \pm 0.33$ & $500-750 \mathrm{mg} 12$ hourly & $1-2$ liters/h & \\
\hline NA & NA & NA & initial $1.5 \mathrm{~g}$, then $750 \mathrm{mg} 20-24$ hourly & mean \pm SD: $0.85 \pm 0.11$ liter $/ \mathrm{h}$ & not critically ill \\
\hline NA & NA & $0.86 \pm 0.08$ & $500 \mathrm{mg} 12$ hourly & $1-2$ liters/h & \\
\hline NA & $0.69 \pm 0.18$ & NA & $\begin{array}{l}2 \mathrm{~g} 8 \text { hourly }(\mathrm{MIC}<4 \mathrm{mg} / \mathrm{l}) \\
3 \mathrm{~g} 8 \text { hourly }(\mathrm{MIC}=8 \mathrm{mg} / \mathrm{l})\end{array}$ & mean \pm SD: $2.82 \pm 0.42$ liters $/ \mathrm{h}$ & \\
\hline $\begin{array}{l}\text { AN69: } 0.97 \pm 0.11, \text { PMMA: } \\
0.80 \pm 0.19, \text { polysulfone: } 0.97 \pm 0.13 \\
\text { (no replacement fluid given) }\end{array}$ & & NS & initial $1 \mathrm{~g}$, then $250-500 \mathrm{mg} 12$ hourly & $\begin{array}{l}\text { CVVH 0.5-1 liter/h } \\
\text { CVVHD 0.5-2 liters/h }\end{array}$ & \\
\hline NA & NA & $0.81 \pm 0.11$ & $\begin{array}{l}\text { initial } 2 \mathrm{~g} \text {, then } 3 \mathrm{~g} \text { daily by continuous } \\
\text { infusion }\end{array}$ & 2.5 liters $/ \mathrm{h}$ & predilution \\
\hline NA & NA & 0.9 & NS & $1.5-2$ liters $/ \mathrm{h}$ & predilution \\
\hline $\begin{array}{l}0.87 \pm 0.46 \\
\text { route of dilution NS }\end{array}$ & NA & NS & mean $\pm \mathrm{SD} 25 \pm 7 \mathrm{ml} / \mathrm{kg} / \mathrm{h}$ & & \\
\hline NA & $0.69 \pm 0.39$ & NA & 2 g daily & $1.2-1.8$ liters $/ \mathrm{h}$ & ceftriaxone not \\
\hline $\begin{array}{l}0.48 \pm 0.13 \text {; } \\
\text { no replacement fluid administered }\end{array}$ & & NA & NS & $0.5-1$ liter/h & $\begin{array}{l}\text { recommended for } \\
\text { patients given }\end{array}$ \\
\hline $\begin{array}{l}0.86 \pm 0.33 \text {; } \\
\text { no replacement fluid administered }\end{array}$ & & NA & NS & $0.5-1$ liter $/ \mathrm{h}$ & $\begin{array}{l}\text { calcium-containing } \\
\text { i.v. solutions }\end{array}$ \\
\hline $\begin{array}{l}0.82 \pm 0.22 \text {; } \\
\text { no replacement fluid administered }\end{array}$ & & NA & NS & $0.5-1$ liter/h & \\
\hline 0.62 & NA & NA & $2 \mathrm{~g} 8$ hourly & $1.0-2.1$ liters $/ \mathrm{h}$ & \\
\hline NA & NA & AN69 0.83, PS 0.97 & 2 g 8 hourly & $2.14-2.5$ liters $/ \mathrm{h}$ & predilution \\
\hline NA & $0.86 \pm 0.04$ & NA & 2-4 g daily & $0.54-1.14$ liters $/ \mathrm{h}$ & \\
\hline NA & NA & $0.78 \pm 0.10$ & 2-4 g daily & $1.78-2.35$ liters $/ \mathrm{h}$ & \\
\hline
\end{tabular}

\begin{tabular}{|c|c|c|c|c|}
\hline NA & $1.21 \pm 0.11$ & NA & $\begin{array}{l}1-1.5 \mathrm{~g} / \text { day }(\mathrm{MIC} \leq 2 \mathrm{mg} / \mathrm{l}) \\
\geq 2 \mathrm{~g} / \text { day }(\text { MIC } 4-8 \mathrm{mg} / \mathrm{l})\end{array}$ & $0.78-1.44$ liters $/ \mathrm{h}$ \\
\hline NA & NA & $1.28 \pm 0.17$ & $\begin{array}{l}1-1.5 \mathrm{~g} / \text { day }(\mathrm{MIC} \leq 2 \mathrm{mg} / \mathrm{l}) \\
\geq 2 \mathrm{~g} / \text { day }(\text { MIC } 4-8 \mathrm{mg} / \mathrm{l})\end{array}$ & $2.0-2.4$ liters $/ \mathrm{h}$ \\
\hline $1.16 / 0.7 ;$ route of dilution & NS & NA & NS & $0.24-7.9$ liters $/ \mathrm{h}$ \\
\hline $\begin{array}{l}\text { imipenem: } 1.2 \pm 0.1 \\
\text { cilastatin: } 0.8 \pm 0.2 \text {; route of dilution }\end{array}$ & NS & NA & $0.5 \mathrm{~g}$ daily & $1.1-1.2$ liters $/ \mathrm{h}$ \\
\hline
\end{tabular}


Table 3 (continued)

\begin{tabular}{|c|c|c|c|c|c|}
\hline Drug (ref. No.) & $\begin{array}{l}\text { Mode of CRRT } \\
\text { (number of } \\
\text { patients) }\end{array}$ & Residual renal function & $\mathrm{Vd}$ & Non-CRRT clearance ${ }^{1}$ & $\begin{array}{l}\text { Membrane/ } \\
\text { surface area }\end{array}$ \\
\hline Imipenem-cilastatin [111] & CVVHD (6) & anuric & $\begin{array}{l}\text { imipenem: } 0.37 \pm 0.16 \text { liter } / \mathrm{kg} \\
\text { cilastatin: } 0.26 \pm 0.09 \text { liter } / \mathrm{kg}\end{array}$ & $\begin{array}{l}\text { imipenem: } 70.6 \pm 18.1 \\
\text { cilastatin: } 18.0 \pm 9.9\end{array}$ & $\mathrm{PAN} / 0.5 \mathrm{~m}^{2}$ \\
\hline Imipenem-cilastatin [112] & $\begin{array}{l}\text { CAVH or } \\
\text { CAVHDF (8) }\end{array}$ & NS & NS & NS & AN69/0.6 m² \\
\hline Imipenem [113] & CVVH (7) & NS & $0.33 \pm 0.09$ liter $/ \mathrm{kg}$ & NI & $\mathrm{PS} / 0.25 \mathrm{~m}^{2}$ \\
\hline Meropenem [13] & CVVH (9) & anuric & $0.36 \pm 0.07$ liter $/ \mathrm{kg}$ & $94.0 \pm 26.9$ & $\mathrm{PS} / 0.43 \mathrm{~m}^{2}$ \\
\hline Meropenem [14] & CVVHDF (9) & anuric & $0.26 \pm 0.09$ liter $/ \mathrm{kg}$ & 22.7 & AN69/0.9 $\mathrm{m}^{2}$ \\
\hline Meropenem [114] & CVVH (9) & $\mathrm{Cl}_{\mathrm{cr}}=1.3 \mathrm{ml} / \mathrm{min}$ & $12.4 \pm 1.8$ liters & $29.9 \pm 5.4$ & AN69/NS \\
\hline Meropenem [15] & CVVH (5) & NS & $0.38 \pm 0.12$ liter $/ \mathrm{kg}$ & $\mathrm{NI}$ & AN69/0.9 $\mathrm{m}^{2}$ \\
\hline Meropenem [15] & CVVHDF (5) & NS & $0.31 \pm 0.08$ liter $/ \mathrm{kg}$ & NI & AN69/0.9 $\mathrm{m}^{2}$ \\
\hline Meropenem [16] & CVVH (8) & $\mathrm{U} / \mathrm{O}<500 \mathrm{ml} / 24 \mathrm{~h}$ & $0.28 \pm 0.07$ liter $/ \mathrm{kg}$ & $58.52 \pm 24.46$ & AN69/0.9 $\mathrm{m}^{2}$ \\
\hline Meropenem [115] & CVVHDF (7) & $\mathrm{Cl}_{\mathrm{cr}}=1.14 \mathrm{ml} / \mathrm{min}$ & $0.57 \pm 0.29$ liter $/ \mathrm{kg}$ & 123.26 & $\begin{array}{l}\text { AN } 69 / 1.4 \mathrm{~m}^{2} \\
\text { PS } / 0.9 \mathrm{~m}^{2}\end{array}$ \\
\hline Meropenem [115] & CVVH (4) & $\mathrm{Cl}_{\mathrm{cr}}=12.5 \pm 12.1 \mathrm{ml} / \mathrm{min}$ & $0.38 \pm 0.07$ liter $/ \mathrm{kg}$ & $114.38 \pm 51.78$ & $\begin{array}{l}\text { AN } 69 / 1.4 \mathrm{~m}^{2} \\
\text { PS } / 0.9 \mathrm{~m}^{2}\end{array}$ \\
\hline Meropenem [115] & CVVHDF (3) & $\mathrm{Cl}_{\mathrm{cr}}=24.8 \pm 18.1 \mathrm{ml} / \mathrm{min}$ & $0.36 \pm 0.14$ liter $/ \mathrm{kg}$ & $85.92 \pm 75.83$ & $\begin{array}{l}\text { AN } 69 / 1.4 \mathrm{~m}^{2} \\
\text { PS } / 0.9 \mathrm{~m}^{2}\end{array}$ \\
\hline Meropenem [116] & CVVHDF (6) & NS & $\begin{array}{l}\text { median (IQR) } \\
32.3 \text { liters (28.9-40.7) }\end{array}$ & NS & $\mathrm{PS} / 1.4 \mathrm{~m}^{2}$ \\
\hline Meropenem [116] & CVVHDF (6) & NS & NS & NS & $\mathrm{PS} / 1.4 \mathrm{~m}^{2}$ \\
\hline Meropenem [18] & CVVH (5) & $\begin{array}{l}1 \text { patient anuric } \\
4 \text { patients } \mathrm{U} / \mathrm{O}<50 \mathrm{ml} / 24 \mathrm{~h}\end{array}$ & $0.37 \pm 0.15$ liter $/ \mathrm{kg}$ & $59 \pm 17.7$ & PAN/0.6 m² \\
\hline Meropenem [117] & CVVHDF (12) & $\begin{array}{l}10 \text { patients anuric } \\
2 \text { patients } \mathrm{U} / \mathrm{O}<180 \mathrm{ml} / 24 \mathrm{~h}\end{array}$ & $0.49 \pm 0.16$ liter $/ \mathrm{kg}$ & $46.96 \pm 29.61$ & AN69/0.9 m² \\
\hline Meropenem [118] & CVVH (10) & oliguric or anuric & $\begin{array}{l}\text { median (IQR) } 0.37 \text { liter } / \mathrm{kg} \\
(0.32-0.46)\end{array}$ & $\begin{array}{l}\text { median (IQR) } 40 \\
(26.7-50.0)\end{array}$ & AN69/2.15 m² \\
\hline Doripenem [119] & CVVHDF (6) & $\mathrm{Cl}_{\mathrm{cr}}: 9.0 \pm 4.1 \mathrm{ml} / \mathrm{min}$ & $0.59 \pm 0.33$ liter $/ \mathrm{kg}$ & 44.5 & $\mathrm{PS} / 1.0 \mathrm{~m}^{2}$ \\
\hline Biapenem [120] & CVVHDF (9) & NS & $0.50 \pm 0.23$ liter $/ \mathrm{kg}$ & $150.8 \pm 67.5$ & PMMA/NS \\
\hline Biapenem [121] & CVVHDF (7) & NS & $10.8 \pm 2.9$ liters (central compartment) & $102.3 \pm 31.5$ & PMMA/NS \\
\hline \multicolumn{6}{|l|}{ Ertapenem (no data) } \\
\hline $\begin{array}{l}\text { Quinolones } \\
\text { Moxifloxacin [122] }\end{array}$ & CVVHDF (9) & anuric & $270 \pm 133$ liters & 291 & AN69/0.9 $\mathrm{m}^{2}$ \\
\hline Levofloxacin [27] & CVVH (12) & anuric & $4.3 \pm 1.8$ liters $/ \mathrm{kg}$ & NS & $\mathrm{PA} / 0.7 \mathrm{~m}^{2}$ \\
\hline Levofloxacin [26] & CVVH (4) & $\begin{array}{l}1 \text { patient anuric } \\
3 \text { patients } \mathrm{U} / \mathrm{O} \leq 40 \mathrm{ml} / 24 \mathrm{~h}\end{array}$ & 1.05 liter $/ \mathrm{kg}$ & 30.8 & $\mathrm{AN} 69 / 0.6 \mathrm{~m}^{2}$ \\
\hline Levofloxacin [26] & CVVHDF (6) & $\mathrm{U} / \mathrm{O} \leq 128 \mathrm{ml} / 24 \mathrm{~h}$ & 1.0 liters $/ \mathrm{kg}$ & 29.5 & AN69/0.6 $\mathrm{m}^{2}$ \\
\hline Levofloxacin [25] & CVVHDF (6) & $\mathrm{Cl}_{\mathrm{cr}}<10 \mathrm{ml} / \mathrm{min}(6)$ & $1.51 \pm 0.52$ liters $/ \mathrm{kg}$ & $28.0 \pm 33.7$ & AN69/0.9 $\mathrm{m}^{2}$ \\
\hline Levofloxacin [25] & CVVH (6) & $\mathrm{Cl}_{\mathrm{cr}}<10 \mathrm{ml} / \mathrm{min}$ & $1.42 \pm 0.42$ liters $/ \mathrm{kg}$ & $32.2 \pm 27.5$ & AN69/0.9 $\mathrm{m}^{2}$ \\
\hline Levofloxacin [28] & CVVH (4) & anuric & $1.02 \pm 0.66$ liters $/ \mathrm{kg}$ & $26.3 \pm 14.8$ & AN69/0.9 $\mathrm{m}^{2}$ \\
\hline Ciprofloxacin [26] & CVVH (5) & $\begin{array}{l}1 \text { patient anuric } \\
4 \text { patients } \mathrm{U} / \mathrm{O} \leq 155 \mathrm{ml} / 24 \mathrm{~h}\end{array}$ & 1.12 liters $/ \mathrm{kg}$ & 72 & AN69/0.6 m ${ }^{2}$ \\
\hline Ciprofloxacin [26] & CVVHDF (5) & $\begin{array}{l}2 \text { patients anuric } \\
3 \text { patients } \mathrm{U} / \mathrm{O} \leq 90 \mathrm{ml} / 24 \mathrm{~h}\end{array}$ & 0.96 liter $/ \mathrm{kg}$ & 125.2 & AN69/0.6 m² \\
\hline Ciprofloxacin [40] & CVVHDF (6) & NS & $1.56 \pm 0.35$ liters $/ \mathrm{kg}$ & NI & AN69/NS \\
\hline Ciprofloxacin [93] & CVVH (16) & $\mathrm{U} / \mathrm{O}<400 \mathrm{ml} / 12 \mathrm{~h}$ & NS & NS & $\mathrm{CT} / 1.9 \mathrm{~m}^{2}$ \\
\hline Ciprofloxacin [123] & CVVHDF (1) & NS & NS & NS & $\mathrm{PA} / 0.6 \mathrm{~m}^{2}$ \\
\hline \multicolumn{6}{|l|}{ Glycopeptides } \\
\hline Vancomycin [124] & CVVH (5) & end-stage renal failure & NA & NS & $\begin{array}{l}\text { AN69/0.6 m } \\
\text { PMMA/ } 2.1 \mathrm{~m}^{2} \\
\text { PS/0.65 m }{ }^{2}\end{array}$ \\
\hline Vancomycin [125] & CVVHDF (10) & NS & $49.7 \pm 29.1$ liters & NI & AN69/NS \\
\hline Vancomycin [126] & CVVH (10) & anuric & $0.55 \pm 0.12$ liter $/ \mathrm{kg}$ & $16.2 \pm 7.0$ (range $3.8-23.3$ ) & $\mathrm{PS} / 0.25 \mathrm{~m}^{2}$ \\
\hline Vancomycin [127] & CVVH (2) & $\mathrm{U} / \mathrm{O} \leq 46 \mathrm{ml} / 24 \mathrm{~h}$ & 41.7 liters, 55.8 liters & 10 & PAN/0.6 m² \\
\hline Vancomycin [128] & CVVH (7) & NS & NS & NS & AN69/1.6 m ${ }^{2}$ \\
\hline Teicoplanin [129] & CVVHDF (3) & $\mathrm{Cl}_{\mathrm{cr}}=2.41 \mathrm{ml} / \mathrm{min}$ & $1.23 \pm 0.77$ liters $/ \mathrm{kg}$ & 7.84 & NS \\
\hline Teicoplanin [130] & CVVH (1) & $\mathrm{Cl}_{\mathrm{cr}}=35 \mathrm{ml} / \mathrm{min}$ & NS & NS & AN69/0.9 m ${ }^{2}$ \\
\hline Teicoplanin [131] & CVVHD (5) & $\mathrm{Cl}_{\mathrm{cr}} 5.8 \pm 2.7 \mathrm{ml} / \mathrm{min}$ & $0.93 \pm 0.42$ liter $/ \mathrm{kg}$ & NS & AN69/0.6 m ${ }^{2}$ \\
\hline Teicoplanin [132] & CVVH (11) & $\mathrm{U} / \mathrm{O} 545 \pm 502 \mathrm{ml} / 24 \mathrm{~h}$ & $0.84 \pm 0.22$ liter $/ \mathrm{kg}$ & $33.7 \mathrm{ml} / \mathrm{kg} / \mathrm{h}$ & $\mathrm{PS} / 1.2 \mathrm{~m}^{2}$ \\
\hline Teicoplanin [132] & CVVH (4) & $\mathrm{U} / \mathrm{O} 545 \pm 502 \mathrm{ml} / 24 \mathrm{~h}$ & $0.48 \pm 0.09$ liter $/ \mathrm{kg}$ & $5.0 \mathrm{ml} / \mathrm{kg} / \mathrm{h}$ & $\mathrm{PS} / 1.2 \mathrm{~m}^{2}$ \\
\hline
\end{tabular}




\begin{tabular}{|c|c|c|c|c|c|}
\hline \multicolumn{2}{|l|}{$S_{c}$} & \multirow[t]{2}{*}{$\mathrm{S}_{\mathrm{d}}$} & \multirow[t]{2}{*}{ Dose recommended by authors } & \multirow{2}{*}{$\begin{array}{l}\text { Total effluent rate (range, } \\
\text { unless otherwise specified) }\end{array}$} & \multirow[t]{2}{*}{ Remarks } \\
\hline pre & post & & & & \\
\hline NA & NA & NS & $\begin{array}{l}0.5 \mathrm{~g} \text { each of imipenem and cilastatin } \\
12 \text { hourly }\end{array}$ & $1.26-1.38$ liters $/ \mathrm{h}$ & \\
\hline $\begin{array}{l}\text { imipenem: } 1.05 \pm 0.19 \\
\text { cilastatin: } 0.68 \pm 0.08\end{array}$ & NA & NS & $0.5 \mathrm{~g} 12$ hourly & $1-3$ liters $/ \mathrm{h}$ & \\
\hline route of dilution & NS & NA & $0.5 \mathrm{~g} 6-8$ hourly & 1 liter/h & \\
\hline NA & $1.09 \pm 0.10$ & NA & $1 \mathrm{~g} 8$ hourly & mean \pm SD: $2.7 \pm 0.4$ liters $/ \mathrm{h}$ & $\begin{array}{l}\text { first-dose pharmaco- } \\
\text { kinetics }\end{array}$ \\
\hline NA & NA & NS & $1 \mathrm{~g} 12$ hourly & 1.6-1.9 liters/h & \\
\hline $1.17 \pm 0.11 ;$ route of dilution & NS & NA & $1 \mathrm{~g}$ daily & $1.1-1.15$ liters $/ \mathrm{h}$ & \\
\hline NA & $0.95 \pm 0.03$ & NA & $1 \mathrm{~g} 12$ hourly & $1.0-2.0$ liters $/ \mathrm{h}$ & \\
\hline NA & NA & $0.92 \pm 0.08$ & $1 \mathrm{~g} 12$ hourly & $2.0-3.0$ liters $/ \mathrm{h}$ & \\
\hline $0.91 \pm 0.10$ & & NA & $500 \mathrm{mg} 12$ hourly & 1.6 liters/h & \\
\hline NA & NA & $\begin{array}{l}\text { AN69 } 0.76 \pm 0.15 \\
\text { PS } 0.76 \pm 0.08\end{array}$ & NS & $1.5-2.5$ liters $/ \mathrm{h}$ & predilution \\
\hline $\begin{array}{l}\text { AN69 } 0.80 \pm 0.15 \text {, } \\
\text { PS } 1.01\end{array}$ & NA & NA & NS & $2.0-2.5$ liters $/ \mathrm{h}$ & \\
\hline NA & NA & $\begin{array}{l}\text { AN69 } 0.82 \pm 0.14 \\
\text { PS } 0.9\end{array}$ & NS & $2.0-2.8$ liters $/ \mathrm{h}$ & predilution \\
\hline NA & NA & $\begin{array}{l}\text { median (IQR) } 0.97 \\
(0.87-1.05)\end{array}$ & $\begin{array}{l}\text { initial } 0.5 \mathrm{~g} \text {, then } 2 \mathrm{~g} \text { over } 24 \mathrm{~h} \\
\text { by continuous infusion }\end{array}$ & $1.4-2.4$ liters $/ \mathrm{h}$ & $\begin{array}{l}\text { first-dose pharmaco- } \\
\text { kinetics }\end{array}$ \\
\hline NA & NA & $\begin{array}{l}\text { median (IQR) } 0.89 \\
(0.79-0.93)\end{array}$ & $\begin{array}{l}\text { initial } 0.5 \mathrm{~g} \text {, then } 2 \mathrm{~g} \text { over } 24 \mathrm{~h} \\
\text { by continuous infusion }\end{array}$ & 1.4-2.4 liters/h & \\
\hline $0.63 \pm 0.25 ;$ route of dilution & NS & NA & $0.5 \mathrm{~g} 12$ hourly & $1.5-1.8$ liters $/ \mathrm{h}$ & \\
\hline NA & NA & $0.65 \pm 0.25$ & $750 \mathrm{mg} 8$ hourly or $1.5 \mathrm{~g} 12$ hourly & $1.11-2.55$ liters $/ \mathrm{h}$ & predilution \\
\hline 0.93 & NA & NA & $1 \mathrm{~g} 8$ hourly & $\begin{array}{l}\text { median (IQR) } 3.5 \\
(3.4-3.9) \text { liters/h }\end{array}$ & predilution \\
\hline NA & NA & NS & $250 \mathrm{mg} 12$ hourly & 0.8 liter $/ \mathrm{h}$ & postdilution \\
\hline NA & NA & $0.92 \pm 0.10$ & $300 \mathrm{mg} 8$ hourly & 1.4 liters $/ \mathrm{h}$ & postdilution \\
\hline NA & NA & $0.92 \pm 0.06$ & $300-600 \mathrm{mg} 12$ hourly & 1.4 liters $/ \mathrm{h}$ & postdilution \\
\hline NA & NA & $0.84 \pm 0.16$ & $400 \mathrm{mg}$ daily & 2 liters/h & predilution \\
\hline $0.47 \pm 0.27 ;$ route of dilution & NS & NA & NS & mean \pm SD: $3.2 \pm 0.9$ liters $/ \mathrm{h}$ & \\
\hline NA & 0.62 & NA & $250 \mathrm{mg} 24$ hourly or $500 \mathrm{mg} 48$ hourly & $0.8-1.3$ liters $/ \mathrm{h}$ & \\
\hline NA & NA & 0.61 & $\begin{array}{l}250 \mathrm{mg} 24 \text { hourly } \\
500 \mathrm{mg} 48 \text { hourly }\end{array}$ & 2-2.4 liters/h & postdilution \\
\hline NA & NA & $0.73 \pm 0.14$ & $250 \mathrm{mg}$ daily & mean: 2.2 liters $/ \mathrm{h}$ & predilution \\
\hline $0.79 \pm 0.14$ & NA & NA & 200 mg daily & mean: 1.2 liters $/ \mathrm{h}$ & \\
\hline NA & $0.98 \pm 0.06$ & NA & initial $500 \mathrm{mg}$, then $250 \mathrm{mg}$ daily & 1.3 liters $/ \mathrm{h}$ & \\
\hline NA & NA & 0.67 & $400 \mathrm{mg}$ daily & $0.54-1.26$ liters $/ \mathrm{h}$ & \\
\hline NA & NA & 0.63 & $400 \mathrm{mg}$ daily & 1.84-2.24 liters/h & \\
\hline NA & NA & $0.70 \pm 0.13$ & 300 mg 12 hourly & 3 liters/h & predilution \\
\hline $0.89 \pm 0.35$; route of dilution & NS & NA & NS & mean \pm SD: $27 \pm 5 \mathrm{ml} / \mathrm{kg} / \mathrm{h}$ & \\
\hline NA & NA & mean \pm SEM: $0.5 \pm 0.067$ & NS & mean: 1.91 liters $/ \mathrm{h}$ & \\
\hline $0.71 \pm 0.19$ & NA & NA & NS & mean \pm SD: $32 \pm 9 \mathrm{ml} / \mathrm{kg} / \mathrm{h}$ & \\
\hline $\begin{array}{l}\text { AN69: } 0.70 \pm 0.15 \\
\text { PMMA: } 0.86 \pm 0.16 \\
\text { PS: } 0.68 \pm 0.19\end{array}$ & & NA & $\begin{array}{l}\text { initial } 15-20 \mathrm{mg} / \mathrm{kg} \text {, then } 0.55-1.25 \mathrm{~g} \\
\text { daily }\left(\mathrm{Cl}_{\mathrm{cr}}<20 \mathrm{ml} / \mathrm{min}\right)\end{array}$ & $0.5-1$ liter $/ \mathrm{h}$ & \\
\hline NA & NA & $0.70 \pm 0.10$ & $450 \mathrm{mg} 12$ hourly & 3 liters/h & predilution \\
\hline NS & NS & NA & NS & $0.5-1.0$ liter/h & \\
\hline NA & $\begin{array}{l}0.88 \pm 0.03 \\
0.89 \pm 0.03\end{array}$ & NA & $\begin{array}{l}\text { initial } 15-20 \mathrm{mg} / \mathrm{kg} \text {, followed after } 24 \mathrm{~h} \\
\text { by } 250-500 \mathrm{mg} 12 \text { hourly }\end{array}$ & 1.5 liters/h & \\
\hline $0.76 \pm 0.11$ & $0.57 \pm 0.15$ & NA & $500 \mathrm{mg} 6$ hourly or $1 \mathrm{~g} 12$ hourly & 6 liters/h & high-volume CVVH \\
\hline NA & NA & NS & NS & NS & \\
\hline $0.13-0.17$ & NA & NA & NS & $1-2$ liters/h & \\
\hline NA & NA & NS & $\begin{array}{l}800 \mathrm{mg} \text { on day } 1 ; 400 \mathrm{mg} \text { on days } 2 \text { and } \\
3, \text { then } 400 \mathrm{mg} 48-72 \text { hourly }\end{array}$ & 0.96 liter/h & \\
\hline $\begin{array}{l}0.14 \pm 0.03(\mathrm{n}=2, \text { predilution; } \\
\mathrm{n}=9, \text { mixed dilution })\end{array}$ & & NA & $1.2 \mathrm{~g}$ of loading dose, $0.6-1.8 \mathrm{~g}$ daily & $35 \mathrm{ml} / \mathrm{kg} / \mathrm{h}(2.4 \pm 0.3$ liters $/ \mathrm{h})$ & $\begin{array}{l}\text { first-dose pharmaco- } \\
\text { kinetics }\end{array}$ \\
\hline $0.17 \pm 0.07$ & NA & NA & $1.2 \mathrm{~g}$ of loading dose, $0.6-1.8 \mathrm{~g}$ daily & $35 \mathrm{ml} / \mathrm{kg} / \mathrm{h}(2.6 \pm 0.1$ liters $/ \mathrm{h})$ & \\
\hline
\end{tabular}


Table 3 (continued)

\begin{tabular}{|c|c|c|c|c|c|}
\hline Drug (ref. No.) & $\begin{array}{l}\text { Mode of CRRT } \\
\text { (number of } \\
\text { patients) }\end{array}$ & Residual renal function & $\mathrm{Vd}$ & Non-CRRT clearance ${ }^{1}$ & $\begin{array}{l}\text { Membrane/ } \\
\text { surface area }\end{array}$ \\
\hline $\begin{array}{l}\text { Aminoglycosides } \\
\text { Gentamicin [133] }\end{array}$ & CAVH (4) & $\begin{array}{l}3 \text { patients } \mathrm{U} / \mathrm{O}<572 \mathrm{ml} / 24 \mathrm{~h} \\
1 \text { patient } \mathrm{U} / \mathrm{O} \text { NS }\end{array}$ & $0.36 \pm 0.09$ liter $/ \mathrm{kg}$ & $9.55 \pm 9.82$ & $\mathrm{PS} / 0.25 \mathrm{~m}^{2}$ \\
\hline Gentamicin [134] & CAVHDF (5) & $\mathrm{Cl}_{\mathrm{cr}}=2.8 \mathrm{ml} / \mathrm{min}$ & NS & $15.26 \pm 7.09$ & $\mathrm{PAN} / 0.43 \mathrm{~m}^{2}$ \\
\hline Netilmicin [135] & CVVHDF (6) & $\mathrm{Cl}_{\mathrm{cr}}=22.3 \pm 6.2 \mathrm{ml} / \mathrm{min}$ & $24.92 \pm 5.96$ liters & NS & AN69/0.6 m² \\
\hline Amikacin [136] & CVVH (5) & anuric & $35 \pm 7.5$ liters & 22.6 & $\mathrm{PS} / 0.6 \mathrm{~m}^{2}$ \\
\hline Amikacin [137] & CVVHDF (6) & NS & $0.47 \pm 0.08$ liter $/ \mathrm{kg}$ & NS & AN69/NS \\
\hline Tobramycin [133] & CAVH (4) & $\begin{array}{l}2 \text { patients anuric. } \\
2 \text { patients } \mathrm{U} / \mathrm{O} 32 \mathrm{ml} / 24 \mathrm{~h}\end{array}$ & $0.28 \pm 0.08$ liter $/ \mathrm{kg}$ & $6.83 \pm 3.22$ & $\mathrm{PS} / 0.25 \mathrm{~m}^{2}$ \\
\hline Arbekacin [138] & CVVHDF (3) & NS & 7.76 liters (central compartment) & NS & PMMA/NS \\
\hline \multicolumn{4}{|l|}{ Miscellaneous } & 37.5 & AN69/NS \\
\hline Linezolid [140] & CVVH (2) & $\mathrm{U} / \mathrm{O}<200 \mathrm{ml} / 24 \mathrm{~h}$ & 0.485 liter $/ \mathrm{kg}$ & NS & $\begin{array}{l}\text { AN69XT/ } \\
1.65 \mathrm{~m}^{2}\end{array}$ \\
\hline Linezolid [141] & CVVH (2) & anuric & 1.02 liters $/ \mathrm{kg}$ & 36.8 & $\mathrm{PS} / 1.25 \mathrm{~m}^{2}$ \\
\hline Linezolid [142] & CVVH (7) & anuric & $0.69 \pm 0.11$ liter $/ \mathrm{kg}$ & $133.5 \pm 71.6$ & $\mathrm{PS} / 1.2 \mathrm{~m}^{2}$ \\
\hline Linezolid [142] & CVVH (13) & anuric & $0.56 \pm 0.14$ liter $/ \mathrm{kg}$ & $118.6 \pm 49.5$ & $\mathrm{PS} / 0.9 \mathrm{~m}^{2}$ \\
\hline Daptomycin [143] & CVVH (10) & in vitro study & NA & NA & $\begin{array}{l}\text { PS/1.5 m² } \\
\text { AN69/0.9 } \mathrm{m}^{2}\end{array}$ \\
\hline Daptomycin [143] & CVVHD (10) & in vitro study & NA & NA & $\begin{array}{l}\text { PS } / 1.5 \mathrm{~m}^{2} \\
\text { AN69/0.9 } \mathrm{m}^{2}\end{array}$ \\
\hline \multicolumn{6}{|c|}{ Clindamycin (no data) } \\
\hline \multicolumn{6}{|c|}{ Rifampicin (no data) } \\
\hline \multicolumn{6}{|c|}{ Azithromycin (no data) } \\
\hline \multicolumn{6}{|c|}{ Clarithromycin (no data) } \\
\hline \multicolumn{6}{|c|}{ Tigecycline (no data) } \\
\hline \multicolumn{6}{|c|}{$\begin{array}{l}\text { Values in parentheses indicate number of patients. Pharmacokinetic data are } \\
\text { steady-state data unless otherwise stated. AN69 = Acrylonitrile; CAVH = continuous } \\
\text { arteriovenous hemofiltration; CAVHD = continuous arteriovenous hemodialysis; } \\
\mathrm{CAVHDF}=\text { continuous arteriovenous hemodiafiltration; } \mathrm{Cl}_{\mathrm{cr}}=\text { creatinine clearance; } \\
\mathrm{Cl}_{\text {urea }}=\text { urea clearance; } \mathrm{CT}=\text { cellulose triacetate; } \mathrm{CVVHD}=\text { continuous venovenous } \\
\text { hemodialysis; } \mathrm{ECMO}=\text { extracorporeal membrane oxygenation; } \mathrm{ESRD}=\text { end-stage re- }\end{array}$} \\
\hline
\end{tabular}

ing clinician's workplace. If this is unavailable, the breakpoint for the organism may be helpful so as to avoid the detrimental consequences of underdosing. Knowledge of published pharmacokinetic data (Vd and non-CRRT clearance) on critically ill patients receiving CRRT, the $\mathrm{S}_{c}$ or $S_{d}$ of the relevant drug (table 3 ) and the dose and mode of CRRT being employed are essential to formulate the dosing regime (refer to examples given in fig. 2a, b). Several aspects of this recommendation require elaboration. First, for the sake of simplicity, the formula recommended for the calculation of the half-life is based on a single compartment and is therefore not strictly accurate. Second, intravenous infusion of antibacterials with time-dependent killing characteristics is recommended because dose estimation is much simpler and not because of any confirmed effect on outcome. If intermittent bolus doses are used the appropriate maintenance dose can be calculated from:
Maintenance dose $=\frac{V d\left(1-e^{-k T}\right) \times C_{t h}}{e^{-k T}}$

and

$$
\text { Loading dose }=\frac{\text { Maintenance dose }}{1-e^{-k T}}
$$

where

$$
k=\frac{C l}{V d},
$$

$\mathrm{T}=$ dosing interval $(\mathrm{min})$ and $\mathrm{C}_{\mathrm{th}}=$ target threshold concentration.

Third, non-CRRT clearance (hepatic and residual renal clearance) needs consideration. Renal clearance needs to be accounted for if residual renal function exists and contributes significantly to total clearance. This is of particular importance if CRRT is being employed for indica- 


\begin{tabular}{|c|c|c|c|c|c|}
\hline \multicolumn{2}{|l|}{$\mathrm{S}_{\mathrm{c}}$} & \multirow[t]{2}{*}{$\mathrm{S}_{\mathrm{d}}$} & \multirow[t]{2}{*}{ Dose recommended by authors } & \multirow{2}{*}{$\begin{array}{l}\text { Total effluent rate (range, } \\
\text { unless otherwise specified) }\end{array}$} & \multirow[t]{2}{*}{ Remarks } \\
\hline pre & post & & & & \\
\hline NA & NA & NA & NS & NS & \\
\hline NA & NA & NS & NS & NS & postdilution \\
\hline NA & NA & NS & $\begin{array}{l}150 \mathrm{mg} 12 \text { hourly does not provide } \\
\text { effective peak levels }\end{array}$ & $0.6-2.2$ liters $/ \mathrm{h}$ & postdilution \\
\hline NS & NS & NA & NI & mean 1.2 liters $/ \mathrm{h}$ & \\
\hline NS & NS & $0.62 \pm 0.2$ & $10 \mathrm{mg} / \mathrm{kg} 48$ hourly & 2 liters/h & \\
\hline NA & NA & NA & NS & NS & \\
\hline NA & NA & 0.74 & $150-200$ mg daily & 1.4 liters $/ \mathrm{h}$ & postdilution \\
\hline NA & NA & NS & $\begin{array}{l}\text { colistin methanesulfonate: } 2-3 \mathrm{mg} / \mathrm{kg} \\
12 \text { hourly }\end{array}$ & 3 liters $/ \mathrm{h}$ & $\begin{array}{l}\text { postdilution data } \\
\text { from a single patient }\end{array}$ \\
\hline 0.57 & NA & NA & NS & $2-2.5$ liters $/ \mathrm{h}$ & \\
\hline 0.84 & NA & NA & NS & 2 liters/h & \\
\hline NA & $0.77 \pm 0.09$ & NA & $600 \mathrm{mg}$ at least 12 hourly & $1.5-3.0$ liters $/ \mathrm{h}$ & \\
\hline NA & $0.69 \pm 0.12$ & NA & $600 \mathrm{mg}$ at least 12 hourly & $1.5-3.0$ liters $/ \mathrm{h}$ & \\
\hline $\begin{array}{l}\text { PS: } 0.16-0.20 \\
\text { AN69: } 0.14-0.16 \\
\end{array}$ & & NA & & 1-6 liters/h & in vitro data \\
\hline NA & NA & $\begin{array}{l}\text { PS: } 0.15 \\
\text { AN69: } 0.05-0.13\end{array}$ & & $1-6$ liters $/ \mathrm{h}$ & in vitro data \\
\hline
\end{tabular}

Table 4. Currently available methods of estimating antibacterial dose in patients receiving CRRT

\begin{tabular}{llll}
\hline Method & Authors & Mode of CRRT & Formula \\
\hline 1 & Bugge [22] & CVVHDF & $D=D_{N} \times\left(P_{x}+\left(1-P_{x}\right)\right) \frac{C l_{C R \text { tot }}}{C l_{C R n}}$ \\
2 & Golper and Marx [23] & CVVH & $D=C_{S S} \times U B F \times U F R \times I$ \\
3 & Schetz et al. [144] & CVVH & $D=D_{N}\left(\frac{C l_{N R}+\left(U F R \times S_{c}\right)}{C l_{N}}\right)$ \\
4 & Schetz et al. [144] & all modes & $D=\frac{D_{\text {anuria }}}{1-\left(\frac{C l_{E C}}{C l_{E C}+C l_{N R}+C l_{R}}\right)}$
\end{tabular}

$\mathrm{C}_{\mathrm{ss}}=$ Measured blood concentration at steady state; $\mathrm{Cl}_{\mathrm{ANUR}}=$ drug clearance in anuric patient; $\mathrm{Cl}_{\mathrm{CRn}}=$ normal creatinine clearance; $\mathrm{Cl}_{\mathrm{CRtot}}=$ sum of renal and extracorporeal creatinine clearance; $\mathrm{Cl}_{\mathrm{EC}}=$ extracorporeal clearance; $\mathrm{Cl}_{\mathrm{N}}=$ normal total drug clearance; $\mathrm{Cl}_{\mathrm{NR}}=$ nonrenal clearance; $\mathrm{Cl}_{\mathrm{R}}=$ renal clearance, $\mathrm{D}_{\text {anuria }}=$ recommended dose for anuric patients; $D_{N}=$ dose recommended for patients with normal renal function; $\mathrm{I}=$ dosing interval; $\mathrm{P}_{\mathrm{x}}=$ extrarenal clearance fraction $\left(=\mathrm{Cl}_{\mathrm{ANUR}} / \mathrm{Cl}_{\mathrm{N}}\right)$; UBF $=$ unbound fraction; UFR = ultrafiltration rate. 
Fig. 1. Calculation of antibacterial doses based on first principles. Non-CRRT clearance is the sum of nonrenal clearance plus residual renal clearance. $\mathrm{Cl}_{\text {tot }}=$ Total clearance.

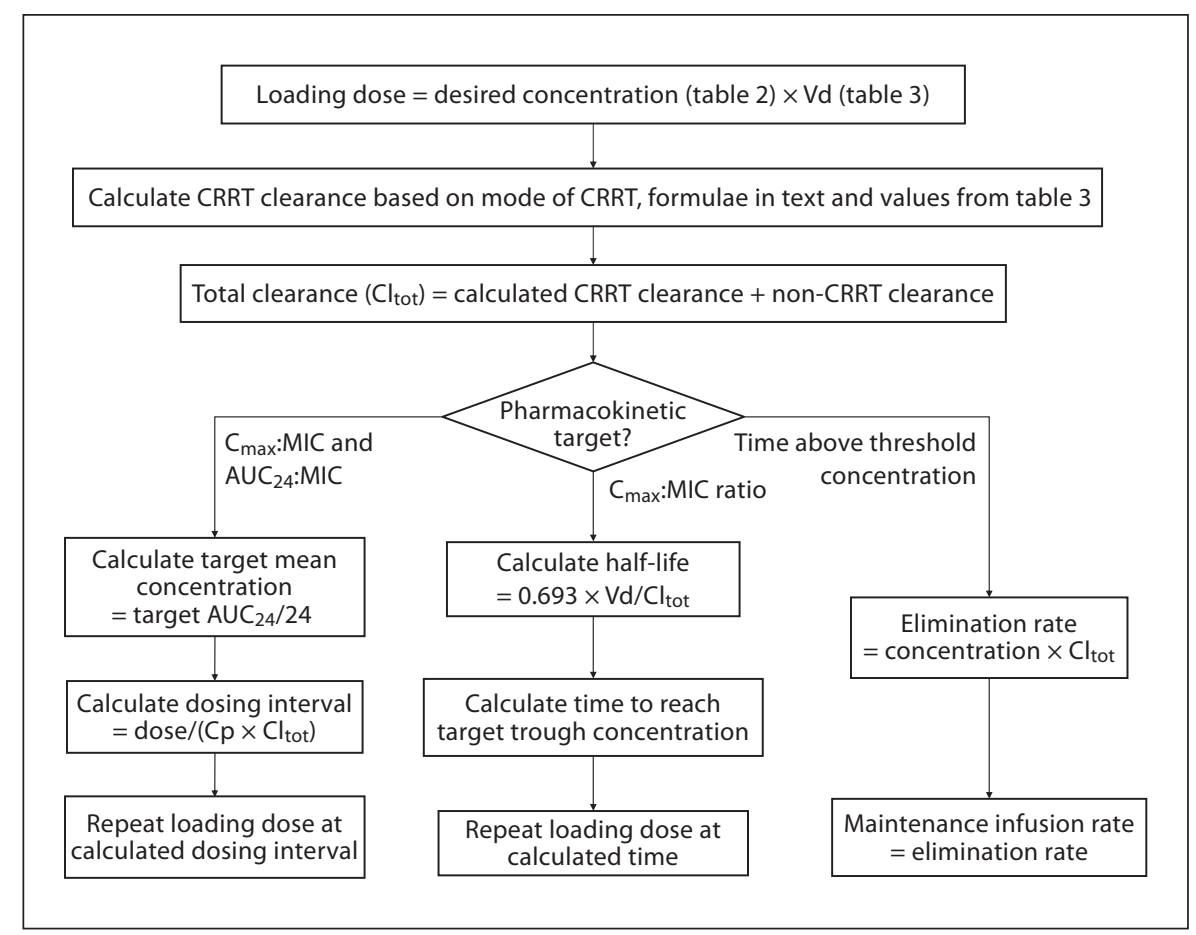

tions other than for AKI (i.e. maintenance of fluid control in massive blood transfusion, dysnatremia and temperature dysregulation). Measurement of creatinine clearance should be considered. In this context, it is important to understand that adjustment for renal clearance based on creatinine clearance assumes that drugs undergo only glomerular filtration and, therefore, this will result in underdosing for drugs with important tubular secretion or overdosing for drugs with tubular reabsorption [10]. Dosing should also take into account the effect of concomitant renal and hepatic failure. For example, the half-life of ciprofloxacin was increased in renal failure, but this was greatly exacerbated by additional hepatic failure [40].

Fourth, when selecting studies from which to obtain $\mathrm{S}_{\mathrm{d}}$, studies which used similar blood and dialysate flows to those being used clinically should be selected. Fifth, if antibacterial concentration assays are available, it is preferable to calculate $S_{c}$ and $S_{d}$ in the individual patient from measured blood and effluent concentrations rather than rely on published values, as individual variability is likely to exist. Sixth, antibacterial doses will need to be adjusted when CRRT doses are altered and if the delivered dose of CRRT differs substantially from the prescribed dose due to interruptions in CRRT.

We believe that our approach to dosing has a number of theoretical advantages. It is, however, important to un- derstand that this method of dosing, like other recommended methods, has not been appropriately validated and like all other methods, it does not take into account inter- and intrapatient pharmacokinetic variability. Furthermore, dosing to achieve the pharmacokinetic targets may result in administration of very large doses, depending on the exact pharmacokinetic target chosen (e.g. time above MIC or time above 5 times MIC) and the MIC. It is important that these doses are prescribed bearing in mind that the benefits of optimal killing need to be balanced against the risks of toxicity and that the possibility of using another agent with a more favorable risk:benefit ratio is considered. Where no suitable alternative exists, it may be prudent to restrict doses to the doses approved by regulatory authorities.

The other major weakness of our approach is the variable quality of reporting of pharmacokinetic data in published studies. A recent review indicated that no reports provided the optimal pharmacokinetic dataset and only $29 \%$ provided a minimum dataset. Even basic data such as $\mathrm{Vd}$ and clearance were missing in approximately $20 \%$ of reports [84]. However, as all dosing methods need to be based on these pharmacokinetic data, this weakness applies to all methods.

It is also important to understand that all the dosing recommendations provided, both old and new, give only 
Fig. 2. a Calculation of amikacin dose for empirical non-Enterobacteriaceae nosocomial sepsis for a $70-\mathrm{kg}$ patient with residual renal function of $15 \mathrm{ml} / \mathrm{min}$ on CVVHDF using an AN69 filter and with targeted total effluent of $35 \mathrm{ml} / \mathrm{kg} / \mathrm{h}$. Note that figures are included for illustrative purposes. The dose prescribed should also take into account the risk of toxicity and may need to be reduced to comply with the dose range approved by the regulatory authorities. b Calculation of the dose of meropenem for empirical non-Enterobacteriaceae/Enterobacteriaceae/Staphylococcus nosocomial sepsis for a $70-\mathrm{kg}$ patient with residual renal function of $20 \mathrm{ml} / \mathrm{min}$ on CVVH (postdilution) using an AN69 $0.9-\mathrm{m}^{2}$ filter with a targeted ultrafiltration rate of $35 \mathrm{ml} / \mathrm{kg} / \mathrm{h}$. Note that figures are included for illustrative purposes. A formula for dose calculation for bolus dosing is given in the text. The dose prescribed should also take into account the risk of toxicity and may need to be reduced to comply with the dose range approved by the regulatory authorities. $\mathrm{Cl}_{\text {tot }}=$ Total clearance; $\mathrm{Cl}_{\mathrm{HF}}=$ hemofiltration clearance; $\mathrm{Cl}_{\mathrm{CVVH}}=\mathrm{CVVH}$ clearance.
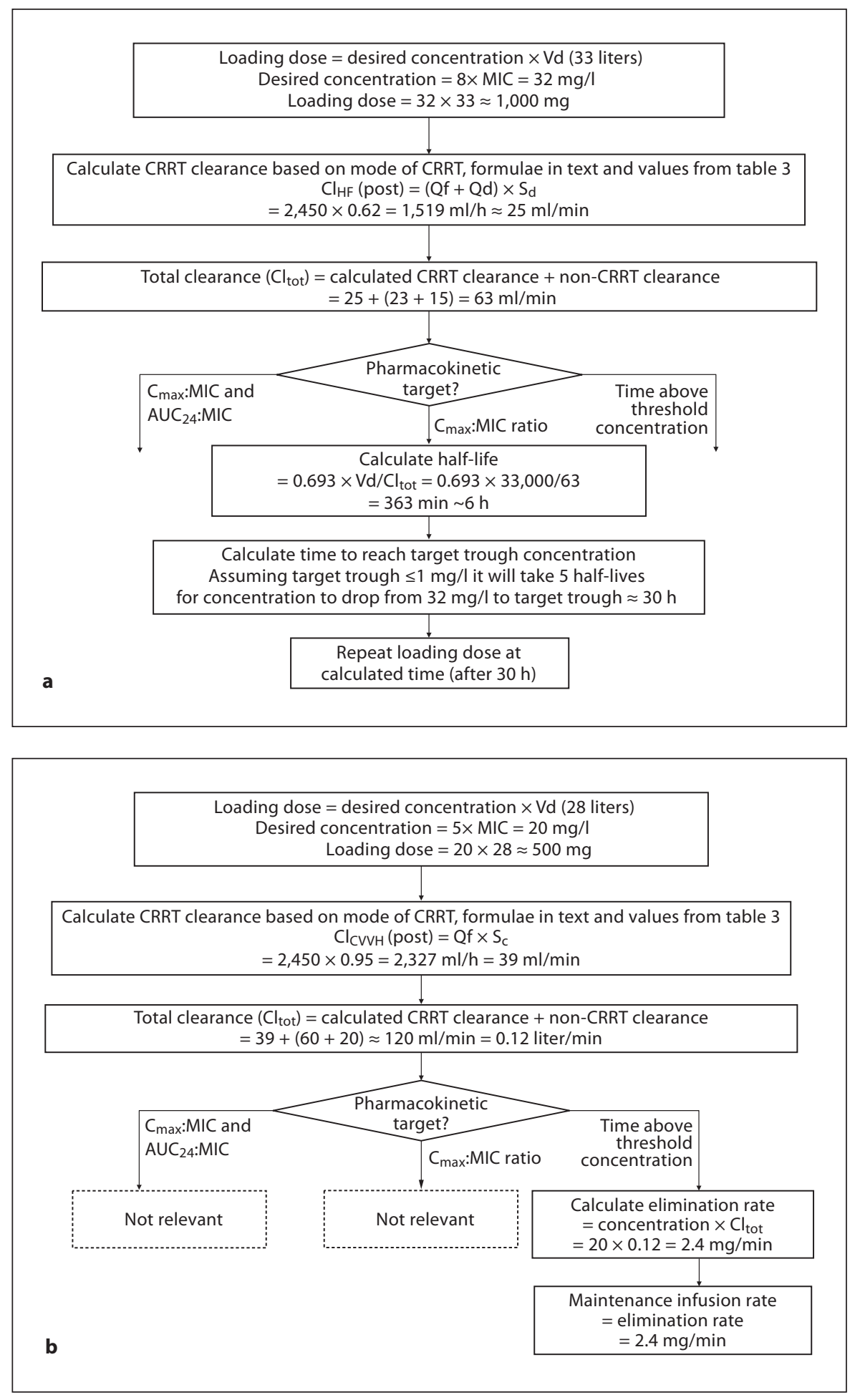
Table 5. A complete dataset recommended for the pharmacokinetic study of patients with antibacterials receiving CRRT

\begin{tabular}{ll}
\hline Category & Parameters \\
\hline $\begin{array}{l}\text { Patient } \\
\text { characteristics }\end{array}$ & $\begin{array}{l}\text { Age, weight, residual renal function, hepatic } \\
\text { function, and severity of illness }\end{array}$ \\
\hline Drug data & Target concentration and dose recommendation \\
\hline $\begin{array}{l}\text { Pharmaco- } \\
\text { kinetics }\end{array}$ & $\begin{array}{l}\text { Vd, protein binding, serum albumin, total clear- } \\
\text { ance, CRRT clearance and non-CRRT clearance }\end{array}$ \\
\hline CRRT & $\begin{array}{l}\text { Hemofilter membrane material and surface area } \\
\text { CVVH: if predilution, } \mathrm{S}_{\mathrm{c}} \text {, ultrafiltration rate, } \\
\text { blood flow rate, hematocrit, and predilution } \\
\text { replacement rate; if post-dilution, } \mathrm{S}_{\mathrm{c}} \text { and ultra- } \\
\text { filtration rate } \\
\text { CVVHD: } \mathrm{S}_{\mathrm{d}}, \text { dialysate rate } \\
\text { CVVHDF: } \mathrm{S}_{\mathrm{c}} / \mathrm{S}_{\mathrm{d}}, \text { ultrafiltration rate and dialysate } \\
\text { rate }\end{array}$ \\
\hline
\end{tabular}

estimated doses and where possible doses should be further adjusted according to measured blood concentrations. Therapeutic drug monitoring has been recommended not only for more toxic agents such as vancomycin and aminoglycosides, but to increase the likelihood of appropriately high dosing of $\beta$-lactams [89]. Although measured drug concentrations can be used to guide empiric increases or decreases in doses, optimal dose adjustment still requires knowledge of the factors discussed above. For example, a measurement showing that the concentration of a $\beta$-lactam is below the threshold concentration early in the dosing interval is helpful in indicating that a dose increase is required, but knowledge of $\mathrm{Vd}$ and clearance is still necessary in order to make the optimal dose adjustment. However, with an appropriate understanding of the issues therapeutic drug monitoring can be used to enhance the approach that we have advocated. For example, by measuring blood and effluent concentrations, $S_{c}$ or $S_{d}$ and hence CRRT clearance can be calculated, or by measuring blood and urine concentrations and measuring urine volume, renal clearance can be calculated. As this method would provide individualized clearance values, it would eliminate many of the problems resulting from the variability of filters, interpatient differences and inadequacy of data reported in published studies. However, the value and cost-effectiveness of antibiotic drug monitoring have yet to be demonstrated, and these assays are not available to most clinicians at present.

Although for some classes of antibiotics appropriate pharmacokinetic targets have been established, this is not the case for all agents (table 2), and for some drugs that are concentrated in tissues (e.g. macrolides, ketolides and azalides), blood concentrations may not be a useful guide [90]. In these cases our proposed method of calculating an appropriate dose is not useful.

\section{Antibiotic Dosing in the Future}

We have presented a method for the calculation of antibiotic dosing that allows appropriate dose delivery to critically ill patients receiving CRRT. The calculation is based on first principles, but relies on the availability of pharmacokinetic data derived from critically ill patients receiving CRRT. Such data are not universally available, partly because of a paucity of investigative studies, especially for older antibiotics such as amoxicillin, ampicillin, flucloxacillin and gentamicin, but also because published studies have frequently failed to report sufficient pharmacokinetic data [84]. Based on the high rate of incomplete reporting, even for important parameters such as Vd, CRRT drug clearance and protein binding, we are of the opinion that a complete dataset such as that previously recommended (table 5) should be reported in all newly published studies as a basic standard.

Finally, therapeutic drug monitoring may alleviate much of the uncertainty of dosing antibiotics in CRRT.

\section{References}

1 Uchino S, Kellum JA, Bellomo R, Doig GS, Morimatsu H, Morgera S, Schetz M, Tan I, Bouman C, Macedo E, Gibney N, Tolwani A, Ronco C: Acute renal failure in critically ill patients: a multinational, multicenter study. JAMA 2005;294:813-818.

$\checkmark 2$ Bagshaw SM, George C, Bellomo R: Early acute kidney injury and sepsis: a multicentre evaluation. Crit Care 2008;12:R47.
Bagshaw SM, Uchino S, Bellomo R, Morimatsu H, Morgera S, Schetz M, Tan I, Bouman C, Macedo E, Gibney N, Tolwani A, Oudemans-van Straaten HM, Ronco C, Kellum JA: Septic acute kidney injury in critically ill patients: clinical characteristics and outcomes. Clin J Am Soc Nephrol 2007;2: 431-439.

\footnotetext{
4 Roberts JA, Kruger P, Paterson DL, Lipman $\mathrm{J}$ : Antibiotic resistance - what's dosing got to do with it? Crit Care Med 2008;36:24332440.

$\checkmark 5$ Rice TW, Bernard GR: Therapeutic intervention and targets for sepsis. Annu Rev Med 2005;56:225-248.
} 
6 Calandra T, Cohen J: The international sepsis forum consensus conference on definitions of infection in the intensive care unit. Crit Care Med 2005;33:1538-1548.

7 Nduka OO, Parrillo JE: The pathophysiology of septic shock. Crit Care Clin 2009;25:677702, vii.

-8 Parrillo JE: Pathogenetic mechanisms of septic shock. N Engl J Med 1993;328:14711477.

$\checkmark 9$ Pea F, Viale P, Furlanut M: Antimicrobial therapy in critically ill patients: a review of pathophysiological conditions responsible for altered disposition and pharmacokinetic variability. Clin Pharmacokinet 2005;44: 1009-1034.

10 Roberts JA, Lipman J: Antibacterial dosing in intensive care: pharmacokinetics, degree of disease and pharmacodynamics of sepsis. Clin Pharmacokinet 2006;45:755-773.

-11 Joynt GM, Lipman J, Gomersall CD, Young RJ, Wong EL, Gin T: The pharmacokinetics of once-daily dosing of ceftriaxone in critically ill patients. J Antimicrob Chemother 2001;47:421-429.

-12 Novelli A, Adembri C, Livi P, Fallani S, Mazzei T, De Gaudio AR: Pharmacokinetic evaluation of meropenem and imipenem in critically ill patients with sepsis. Clin Pharmacokinet 2005;44:539-549.

13 Thalhammer F, Schenk P, Burgmann H, El Menyawi I, Hollenstein UM, Rosenkranz AR, Sunder-Plassmann G, Breyer S, Ratheiser K: Single-dose pharmacokinetics of meropenem during continuous venovenous hemofiltration. Antimicrob Agents Chemother 1998;42:2417-2420.

-14 Krueger WA, Schroeder TH, Hutchison M, Hoffmann E, Dieterich HJ, Heininger A, Erley C, Wehrle A, Unertl K: Pharmacokinetics of meropenem in critically ill patients with acute renal failure treated by continuous hemodiafiltration. Antimicrob Agents Chemother 1998;42:2421-2424.

15 Giles LJ, Jennings AC, Thomson AH, Creed G, Beale RJ, McLuckie A: Pharmacokinetics of meropenem in intensive care unit patients receiving continuous veno-venous hemofiltration or hemodiafiltration. Crit Care Med 2000;28:632-637.

-16 Krueger WA, Neeser G, Schuster H, Schroeder TH, Hoffmann E, Heininger A, Dieterich $\mathrm{HJ}$, Forst $\mathrm{H}$, Unertl KE: Correlation of meropenem plasma levels with pharmacodynamic requirements in critically ill patients receiving continuous veno-venous hemofiltration. Chemotherapy 2003;49: 280-286.

17 Kielstein JT, Czock D, Schopke T, Hafer C, Bode-Boger SM, Kuse E, Keller F, Fliser D: Pharmacokinetics and total elimination of meropenem and vancomycin in intensive care unit patients undergoing extended daily dialysis. Crit Care Med 2006;34:51-56.
18 Ververs TF, van Dijk A, Vinks SA, Blankestijn PJ, Savelkoul JF, Meulenbelt J, Boereboom FT: Pharmacokinetics and dosing regimen of meropenem in critically ill patients receiving continuous venovenous hemofiltration. Crit Care Med 2000;28:3412-3416.

-19 Hassan E, Ober JD: Predicted and measured aminoglycoside pharmacokinetic parameters in critically ill patients. Antimicrob Agents Chemother 1987;31:1855-1858.

20 Young RJ, Lipman J, Gin T, Gomersall CD, Joynt GM, Oh TE: Intermittent bolus dosing of ceftazidime in critically ill patients. J Antimicrob Chemother 1997;40:269-273.

-21 Isla A, Gascon AR, Maynar J, Arzuaga A, Sanchez-Izquierdo JA, Pedraz JL: In vitro AN69 and polysulphone membrane permeability to ceftazidime and in vivo pharmacokinetics during continuous renal replacement therapies. Chemotherapy 2007;53: 194-201.

22 Bugge JF: Pharmacokinetics and drug dosing adjustments during continuous venovenous hemofiltration or hemodiafiltration in critically ill patients. Acta Anaesthesiol Scand 2001;45:929-934.

23 Golper TA, Marx MA: Drug dosing adjustments during continuous renal replacement therapies. Kidney Int Suppl 1998;66:S165S168.

24 Golper TA: Update on drug sieving coefficients and dosing adjustments during continuous renal replacement therapies. Contrib Nephrol 2001;132:349-353.

$>25$ Guenter SG, Iven H, Boos C, Bruch HP, Muhl E: Pharmacokinetics of levofloxacin during continuous venovenous hemodiafiltration and continuous venovenous hemofiltration in critically ill patients. Pharmacotherapy 2002;22:175-183.

26 Malone RS, Fish DN, Abraham E, Teitelbaum I: Pharmacokinetics of levofloxacin and ciprofloxacin during continuous renal replacement therapy in critically ill patients. Antimicrob Agents Chemother 2001;45: 2949-2954.

-27 Traunmuller F, Thalhammer-Scherrer R, Locker GJ, Losert H, Schmid R, Staudinger T, Thalhammer F: Single-dose pharmacokinetics of levofloxacin during continuous veno-venous haemofiltration in critically ill patients. J Antimicrob Chemother 2001;47: 229-231.

28 Hansen E, Bucher M, Jakob W, Lemberger P, Kees F: Pharmacokinetics of levofloxacin during continuous veno-venous hemofiltration. Intensive Care Med 2001;27:371-375.

29 Meyers BR, Srulevitch ES, Jacobson J, Hirschman SZ: Crossover study of the pharmacokinetics of ceftriaxone administered intravenously or intramuscularly to healthy volunteers. Antimicrob Agents Chemother 1983;24:812-814.

-30 Seddon M, Wise R, Gillett AP, Livingston R: Pharmacokinetics of Ro 13-9904, a broad-spectrum cephalosporin. Antimicrob Agents Chemother 1980;18:240-242.
31 Krueger WA, Schroeder TH, Hansen M: Pharmacokinetics of antibiotics during continuous renal replacement therapy; in Vincent JL (ed): Yearbook of Intensive Care and Emergency Medicine. New York, Springer, 2005, pp 349-359.

32 Ronco C, Bellomo R: Principles of solute clearance during continuous renal replacement therapy; in Ronco C, Bellomo R (eds): Critical Care Nephrology. Dordrecht, Boston Kluwer Academic, 1998, pp 1213-1223.

33 Kubin C, Dzierba A: The effects of continuous renal replacement on anti-infective therapy in the critically ill. J Pharm Pract 2005; 18:109-117.

34 Gonzalez MA, Moranchel AH, Duran S, Pichardo A, Magana JL, Painter B, Drusano GL: Multiple-dose ciprofloxacin dose ranging and kinetics. Clin Pharmacol Ther 1985; 37:633-637.

$\checkmark 35$ Chow AT, Fowler C, Williams RR, Morgan N, Kaminski S, Natarajan J: Safety and pharmacokinetics of multiple 750-milligram doses of intravenous levofloxacin in healthy volunteers. Antimicrob Agents Chemother 2001;45:2122-2125.

$>36$ de Pont AC: Extracorporeal treatment of intoxications. Curr Opin Crit Care 2007;13: 668-673.

\$37 Choi G, Gomersall CD, Lipman J, Wong A, Joynt GM, Leung P, Ramsay SJ, Ho OM: The effect of adsorption, filter material and point of dilution on antibiotic elimination by haemofiltration an in vitro study of levofloxacin. Int J Antimicrob Agents 2004;24:468472.

38 Tian Q, Gomersall CD, Ip M, Tan PE, Joynt GM, Choi GY: Adsorption of amikacin, a significant mechanism of elimination by hemofiltration. Antimicrob Agents Chemother 2008;52:1009-1013.

39 Tian Q, Gomersall CD, Leung PP, Choi GY, Joynt GM, Tan PE, Wong AS: The adsorption of vancomycin by polyacrylonitrile, polyamide, and polysulfone hemofilters. Artif Organs 2008;32:81-84.

40 Wallis SC, Mullany DV, Lipman J, Rickard CM, Daley PJ: Pharmacokinetics of ciprofloxacin in ICU patients on continuous veno-venous haemodiafiltration. Intensive Care Med 2001;27:665-672.

41 Hawker F: Liver dysfunction in critical illness. Anaesth Intensive Care 1991;19:165181.

42 Rolando N, Wade J, Davalos M, Wendon J, Philpott-Howard J, Williams R: The systemic inflammatory response syndrome in acute liver failure. Hepatology 2000;32:734-739.

43 Davis R, Markham A, Balfour JA: Ciprofloxacin. An updated review of its pharmacology, therapeutic efficacy and tolerability. Drugs 1996;51:1019-1074. 
-44 Jones EM, McMullin CM, Hedges AJ, Lovering AM, White LO, Reeves DS, MacGowan AP: The pharmacokinetics of intravenous ciprofloxacin $400 \mathrm{mg} 12$ hourly in patients with severe sepsis: the effect of renal function and intra-abdominal disease. J Antimicrob Chemother 1997;40:121-124.

45 Nicolau DP: Optimizing outcomes with antimicrobial therapy through pharmacodynamic profiling. J Infect Chemother 2003;9: 292-296.

46 Craig WA: Pharmacokinetic/pharmacodynamic parameters: rationale for antibacterial dosing of mice and men. Clin Infect Dis 1998;26:1-10; quiz 11-12.

-47 Burgess DS: Pharmacodynamic principles of antimicrobial therapy in the prevention of resistance. Chest 1999;115:19S-23S.

-48 Rodvold KA: Pharmacodynamics of antiinfective therapy: taking what we know to the patient's bedside. Pharmacotherapy 2001;21: 319S-330S.

49 Turnidge JD: The pharmacodynamics of beta-lactams. Clin Infect Dis 1998;27:10-22.

-50 Drusano GL: Prevention of resistance: a goal for dose selection for antimicrobial agents. Clin Infect Dis 2003;36:S42-S50.

- 51 Fantin B, Farinotti R, Thabaut A, Carbon C: Conditions for the emergence of resistance to cefpirome and ceftazidime in experimental endocarditis due to Pseudomonas aeruginosa. J Antimicrob Chemother 1994;33:563569.

- 52 Leggett JE, Fantin B, Ebert S, Totsuka K, Vogelman B, Calame W, Mattie H, Craig WA: Comparative antibiotic dose-effect relations at several dosing intervals in murine pneumonitis and thigh-infection models. J Infect Dis 1989;159:281-292.

53 Moore RD, Lietman PS, Smith CR: Clinical response to aminoglycoside therapy: importance of the ratio of peak concentration to minimal inhibitory concentration. J Infect Dis 1987; 155:93-99.

-54 Buijk SE, Mouton JW, Gyssens IC, Verbrugh HA, Bruining HA: Experience with a oncedaily dosing program of aminoglycosides in critically ill patients. Intensive Care Med 2002;28:936-942.

- 55 Kashuba AD, Nafziger AN, Drusano GL, Bertino JS Jr: Optimizing aminoglycoside therapy for nosocomial pneumonia caused by gram-negative bacteria. Antimicrob Agents Chemother 1999;43:623-629.

56 Vogelman B, Gudmundsson S, Leggett J, Turnidge J, Ebert S, Craig WA: Correlation of antimicrobial pharmacokinetic parameters with therapeutic efficacy in an animal model. J Infect Dis 1988;158:831-847.

57 Vogelman B, Craig WA: Kinetics of antimicrobial activity. J Pediatr 1986;108:835-840.

- 58 Vogelman BS, Craig WA: Postantibiotic effects. J Antimicrob Chemother 1985; 15 (suppl A):37-46.
59 Roosendaal R, Bakker-Woudenberg IA, van den Berghe-van Raffe M, Vink-van den Berg JC, Michel BM: Impact of the dosage schedule on the efficacy of ceftazidime, gentamicin and ciprofloxacin in Klebsiella pneu moniae pneumonia and septicemia in leukopenic rats. Eur J Clin Microbiol Infect Dis $1989 ; 8: 878-887$.

60 Craig W: Pharmacokinetic and experimental data on beta-lactam antibiotics in the treatment of patients. Eur J Clin Microbio 1984;3:575-578

61 Mouton JW, Vinks AA: Continuous infusion of beta-lactams. Curr Opin Crit Care 2007; 13:598-606.

62 Prins JM, Buller HR, Kuijper EJ, Tange RA, Speelman P: Once versus thrice daily gentamicin in patients with serious infections. Lancet 1993;341:335-339.

63 Marik PE, Havlik I, Monteagudo FS, Lipman J: The pharmacokinetic of amikacin in critically ill adult and paediatric patients: comparison of once- versus twice-daily dosing regimens. J Antimicrob Chemother 1991; 27(suppl C):81-89.

64 Marik PE, Lipman J, Kobilski S, Scribante J: A prospective randomized study comparing once- versus twice-daily amikacin dosing in critically ill adult and paediatric patients. J Antimicrob Chemother 1991;28:753-764.

65 Van der Auwera P, Meunier F, Ibrahim S, Kaufman L, Derde MP, Tulkens PM: Pharmacodynamic parameters and toxicity of netilmicin (6 milligrams/kilogram/day) given once daily or in three divided doses to cancer patients with urinary tract infection. Antimicrob Agents Chemother 1991;35: 640-647.

-66 Preston SL, Drusano GL, Berman AL, Fowler CL, Chow AT, Dornseif B, Reichl V, Natarajan J, Corrado M: Pharmacodynamics of levofloxacin: a new paradigm for early clinical trials. JAMA 1998;279:125-129.

67 Forrest A, Nix DE, Ballow CH, Goss TF, Birmingham MC, Schentag JJ: Pharmacodynamics of intravenous ciprofloxacin in seriously ill patients. Antimicrob Agents Chemother 1993;37:1073-1081.

-68 Arzuaga A, Maynar J, Gascon AR, Isla A, Corral E, Fonseca F, Sanchez-Izquierdo JA, Rello J, Canut A, Pedraz JL: Influence of renal function on the pharmacokinetics of piperacillin/tazobactam in intensive care unit patients during continuous venovenous hemofiltration. J Clin Pharmacol 2005;45: 168-176.

69 Ross GH, Wright DH, Hovde LB, Peterson ML, Rotschafer JC: Fluoroquinolone resistance in anaerobic bacteria following exposure to levofloxacin, trovafloxacin, and sparfloxacin in an in vitro pharmacodynamic model. Antimicrob Agents Chemother 2001; 45:2136-2140.
70 LaPlante KL, Rybak MJ, Tsuji B, Lodise TP, Kaatz GW: Fluoroquinolone resistance in Streptococcus pneumoniae: area under the concentration-time curve/MIC ratio and resistance development with gatifloxacin, gemifloxacin, levofloxacin, and moxifloxacin. Antimicrob Agents Chemother 2007;51: 1315-1320.

71 Schentag JJ: Antimicrobial action and pharmacokinetics/pharmacodynamics: the use of AUIC to improve efficacy and avoid resistance. J Chemother 1999;11:426-439.

72 Selimoglu E: Aminoglycoside-induced ototoxicity. Curr Pharm Des 2007;13:119-126.

73 Drusano GL, Ambrose PG, Bhavnani SM, Bertino JS, Nafziger AN, Louie A: Back to the future: using aminoglycosides again and how to dose them optimally. Clin Infect Dis 2007;45:753-760.

74 Pannu N, Nadim MK: An overview of druginduced acute kidney injury. Crit Care Med 2008;36:S216-S223.

75 Rubinstein E, Camm J: Cardiotoxicity of fluoroquinolones. J Antimicrob Chemother 2002;49:593-596.

76 Chow KM, Hui AC, Szeto CC: Neurotoxicity induced by beta-lactam antibiotics: from bench to bedside. Eur J Clin Microbiol Infect Dis 2005;24:649-653.

-77 Rybak MJ, Lomaestro BM, Rotschafer JC, Moellering RC, Craig WA, Billeter M, Dalovisio JR, Levine DP: Vancomycin therapeutic guidelines: a summary of consensus recommendations from the Infectious Diseases Society of America, the American Society of Health-System Pharmacists, and the Society of Infectious Diseases Pharmacists. Clin Infect Dis 2009;49:325-327.

78 Schliamser SE, Cars O, Norrby SR: Neurotoxicity of beta-lactam antibiotics: predisposing factors and pathogenesis. J Antimicrob Chemother 1991;27:405-425.

79 Lam S, Gomolin IH: Cefepime neurotoxicity: case report, pharmacokinetic considerations, and literature review. Pharmacotherapy 2006;26:1169-1174.

80 Capparelli FJ, Diaz MF, Hlavnika A, Wainsztein NA, Leiguarda R, Del Castillo ME: Cefepime- and cefixime-induced encephalopathy in a patient with normal renal function. Neurology 2005;65:1840

81 Chenel M, Limosin A, Marchand S, Paquereau J, Mimoz O, Couet W: Norfloxacininduced electroencephalogram alteration and seizures in rats are not triggered by enhanced levels of intracerebral glutamate. Antimicrob Agents Chemother 2003;47:36603662

82 Gilbert DN: The Sanford Guide to Antimicrobial Therapy 2006, ed 36. Hyde Park, Antimicrobial Therapy Inc., 2006.

-83 Trotman RL, Williamson JC, Shoemaker DM, Salzer WL: Antibiotic dosing in critically ill adult patients receiving continuous renal replacement therapy. Clin Infect Dis 2005;41:1159-1166 
84 Churchwell MD, Mueller BA: Drug dosing during continuous renal replacement therapy. Semin Dial 2009;22:185-188.

85 Leone M, Albanese J, Sampol-Manos E, Simon N, Lacarelle B, Bruguerolle B, Martin C: Moxifloxacin penetration in bronchial secretions of mechanically ventilated patients with pneumonia. Antimicrob Agents Chemother 2004;48:638-640.

86 Song JH, Jung SI, Ko KS, Kim NY, Son JS, Chang HH, Ki HK, Oh WS, Suh JY, Peck KR, Lee NY, Yang Y, Lu Q, Chongthaleong A, Chiu CH, Lalitha MK, Perera J, Yee TT, Kumarasinghe G, Jamal F, Kamarulzaman A, Parasakthi N, Van PH, Carlos C, So T, Ng TK, Shibl A: High prevalence of antimicrobial resistance among clinical Streptococcus pneumoniae isolates in Asia (an ANSORP study). Antimicrob Agents Chemother 2004; 48:2101-2107.

-87 Ambrose PG, Grasela DM, Grasela TH, Passarell J, Mayer HB, Pierce PF: Pharmacodynamics of fluoroquinolones against Streptococcus pneumoniae in patients with community-acquired respiratory tract infections. Antimicrob Agents Chemother 2001;45:2793-2797.

88 Mueller BA, Pasko DA, Sowinski KM: Higher renal replacement therapy dose delivery influences on drug therapy. Artif Organs 2003;27:808-814.

89 Roberts JA, Hope WW, Lipman J: Therapeutic drug monitoring of beta-lactams for critically ill patients: unwarranted or essential? Int J Antimicrob Agents 2010;35:419-420.

90 Drusano GL: Infection site concentrations: their therapeutic importance and the macrolide and macrolide-like class of antibiotics. Pharmacotherapy 2005;25:150S-158S.

91 Schentag JJ: Clinical pharmacology of the fluoroquinolones: studies in human dynamic/kinetic models. Clin Infect Dis 2000; 31(suppl 2):S40-S44.

92 Andes D, van Ogtrop ML, Peng J, Craig WA: In vivo pharmacodynamics of a new oxazolidinone (linezolid). Antimicrob Agents Chemother 2002;46:3484-3489.

-93 Bouman CS, van Kan HJ, Koopmans RP, Korevaar JC, Schultz MJ, Vroom MB: Discrepancies between observed and predicted continuous venovenous hemofiltration removal of antimicrobial agents in critically ill patients and the effects on dosing. Intensive Care Med 2006;32:2013-2019.

94 Capellier G, CornetteC, Boillot A, Guinchard C, Jacques T, Blasco G, Barale F: Removal of piperacillin in critically ill patients undergoing continuous venovenous hemofiltration. Crit Care Med 1998;26:88-91.

95 Keller E, Bohler J, Busse-Grawitz A, ReetzeBonorden P, Krumme B, Schollmeyer P: Single dose kinetics of piperacillin during continuous arteriovenous hemodialysis in intensive care patients. Clin Nephrol 1995; 43(suppl 1):S20-S23.
$\$ 96$ Mueller SC, Majcher-Peszynska J, Hickstein H, Francke A, Pertschy A, Schulz M, Mundkowski R, Drewelow B: Pharmacokinetics of piperacillin-tazobactam in anuric intensive care patients during continuous venovenous hemodialysis. Antimicrob Agents Chemother 2002;46:1557-1560.

$\$ 97$ Lindsay CA, Bawdon R, Quigley R: Clearance of ticarcillin-clavulanic acid by continuous venovenous hemofiltration in three critically ill children, two with and one without concomitant extracorporeal membrane oxygenation. Pharmacotherapy 1996;16:458-462.

98 Meyer B, Ahmed el Gendy S, Delle Karth G, Locker GJ, Heinz G, Jaeger W, Thalhammer F: How to calculate clearance of highly protein-bound drugs during continuous venovenous hemofiltration demonstrated with flucloxacillin. Kidney Blood Press Res 2003;26:135-140.

$\$ 99$ Davies SP, Lacey LF, Kox WJ, Brown EA: Pharmacokinetics of cefuroxime and ceftazidime in patients with acute renal failure treated by continuous arteriovenous haemodialysis. Nephrol Dial Transplant 1991; 6:971-976.

100 Weiss LG, Cars O, Danielson BG, Grahnen A, Wikstrom B: Pharmacokinetics of intravenous cefuroxime during intermittent and continuous arteriovenous hemofiltration. Clin Nephrol 1988;30:282-286.

101 Traunmuller F, Schenk P, Mittermeyer C, Thalhammer-Scherrer R, Ratheiser K, Thalhammer F: Clearance of ceftazidime during continuous venovenous haemofiltration in critically ill patients. J Antimicrob Chemother 2002;49:129-134.

102 Matzke GR, Frye RF, Joy MS, Palevsky PM: Determinants of ceftazidime clearance by continuous venovenous hemofiltration and continuous venovenous hemodialysis. An timicrob Agents Chemother 2000;44:16391644.

103 Mariat C, Venet C, Jehl F, Mwewa S, Lazarevic V, Diconne E, Fonsale N, Carricajo A, Guyomarc'h S, Vermesch R, Aubert G, Bidault R, Bertrand JC, Zeni F: Continuous infusion of ceftazidime in critically ill patients undergoing continuous venovenous haemodiafiltration: pharmacokinetic evaluation and dose recommendation. Crit Care 2006; 10:R26.

104 Kroh UF, Lennartz H, Edwards DI, Stoeckel K: Pharmacokinetics of ceftriaxone in patients undergoing continuous venovenous hemofiltration. J Clin Pharmacol 1996;36:1114-1119.

105 Matzke GR, Frye RF, Joy MS, Palevsky PM: Determinants of ceftriaxone clearance by continuous venovenous hemofiltration and hemodialysis. Pharmacotherapy 2000;20: 635-643.
106 Isla A, Gascon AR, Maynar J, Arzuaga A, Toral D, Pedraz JL: Cefepime and continuous renal replacement therapy (CRRT): in vitro permeability of two CRRT membranes and pharmacokinetics in four critically ill patients. Clin Ther 2005;27:599608.

107 Malone RS, Fish DN, Abraham E, Teitelbaum I: Pharmacokinetics of cefepime during continuous renal replacement therapy in critically ill patients. Antimicrob Agents Chemother 2001;45:3148-3155.

108 Fish DN, Teitelbaum I, Abraham E: Pharmacokinetics and pharmacodynamics of imipenem during continuous renal replacement therapy in critically ill patients. Antimicrob Agents Chemother 2005;49: 2421-2428.

109 Keller E, Fecht H, Bohler J, Schollmeyer P: Single-dose kinetics of imipenem/cilastatin during continuous arteriovenous haemofiltration in intensive care patients. Nephrol Dial Transplant 1989;4:640-645.

110 Tegeder I, Bremer F, Oelkers R, Schobel H, Schuttler J, Brune K, Geisslinger G: Pharmacokinetics of imipenem-cilastatin in critically ill patients undergoing continuous venovenous hemofiltration. Antimicrob Agents Chemother 1997;41:26402645.

-111 Hashimoto S, Honda M, Yamaguchi M, Sekimoto M, Tanaka Y: Pharmacokinetics of imipenem and cilastatin during continuous venovenous hemodialysis in patients who are critically ill. ASAIO J 1997;43:8488.

112 Vos MC, Vincent HH, Yzerman EP: Clearance of imipenem/cilastatin in acute renal failure patients treated by continuous hemodiafiltration (CAVHD). Intensive Care Med 1992;18:282-285.

113 Mueller BA, Scarim SK, Macias WL: Comparison of imipenem pharmacokinetics in patients with acute or chronic renal failure treated with continuous hemofiltration. Am J Kidney Dis 1993;21:172-179.

114 Tegeder I, Neumann F, Bremer F, Brune K, Lotsch J, Geisslinger G: Pharmacokinetics of meropenem in critically ill patients with acute renal failure undergoing continuous venovenous hemofiltration. Clin Pharmacol Ther 1999;65:50-57.

- 115 Isla A, Maynar J, Sanchez-Izquierdo JA, Gascon AR, Arzuaga A, Corral E, Pedraz JL: Meropenem and continuous renal replacement therapy: in vitro permeability of 2 continuous renal replacement therapy membranes and influence of patient renal function on the pharmacokinetics in critically ill patients. J Clin Pharmacol 2005;45: 1294-1304.

- 116 Langgartner J, Vasold A, Gluck T, Reng M, Kees F: Pharmacokinetics of meropenem during intermittent and continuous intravenous application in patients treated by continuous renal replacement therapy. Intensive Care Med 2008;34:1091-1096. 
-117 Robatel C, Decosterd LA, Biollaz J, Eckert P, Schaller MD, Buclin T: Pharmacokinetics and dosage adaptation of meropenem during continuous venovenous hemodiafiltration in critically ill patients. J Clin Pharmacol 2003;43:1329-1340.

118 Bilgrami I, Roberts JA, Wallis SC, Thomas J, Davis J, Fowler S, Goldrick PB, Lipman J: Meropenem dosing in critically ill patients with sepsis receiving high volume continuous veno-venous hemofiltration. Antimicrob Agents Chemother 2010.

-119 Hidaka S, Goto K, Hagiwara S, Iwasaka H, Noguchi T: Doripenem pharmacokinetics in critically ill patients receiving continuous hemodiafiltration (CHDF). Yakugaku Zasshi 2010;130:87-94.

- 120 Suyama H, Ikawa K, Morikawa N, Ikeda K, Fujiue Y, Morikawa S, Kaneko K, Kuwabara M, Yamanoue T: Pharmacokinetics and pharmacodynamics of biapenem in critically ill patients under continuous venovenous hemodiafiltration. Jpn J Antibiot 2008;61:303-313.

-121 Ikawa K, Morikawa N, Ikeda K, Suyama H: Pharmacokinetic modeling and dosage adaptation of biapenem in Japanese patients during continuous venovenous hemodiafiltration. J Infect Chemother 2008;14:3539.

-122 Fuhrmann V, Schenk P, Jaeger W, Ahmed S, Thalhammer F: Pharmacokinetics of moxifloxacin in patients undergoing continuous venovenous haemodiafiltration. J Antimicrob Chemother 2004;54:780-784.

-123 Barrie JR, Mousdale S: Ciprofloxacin levels in a patient undergoing veno-venous haemodiafiltration. Intensive Care Med 1992; 18:437-438.

-124 Joy MS, Matzke GR, Frye RF, Palevsky PM: Determinants of vancomycin clearance by continuous venovenous hemofiltration and continuous venovenous hemodialysis. Am J Kidney Dis 1998;31:1019-1027.

-125 DelDot ME, Lipman J, Tett SE: Vancomycin pharmacokinetics in critically ill patients receiving continuous venovenous haemodiafiltration. Br J Clin Pharmacol 2004;58: 259-268.
126 Macias WL, Mueller BA, Scarim SK: Vancomycin pharmacokinetics in acute renal failure: preservation of nonrenal clearance. Clin Pharmacol Ther 1991;50:688-694.

127 Boereboom FT, Ververs FF, Blankestijn PJ Savelkoul TJ, van Dijk A: Vancomycin clearance during continuous venovenous haemofiltration in critically ill patients. Intensive Care Med 1999;25:1100-1104.

128 Uchino S, Cole L, Morimatsu H, Goldsmith D, Bellomo R: Clearance of vancomycin during high-volume haemofiltration: impact of pre-dilution. Intensive Care Med 2002;28:1664-1667.

129 Yagasaki K, Gando S, Matsuda N, Kameue T, Ishitani T, Hirano T, Iseki K: Pharmacokinetics of teicoplanin in critically ill patients undergoing continuous hemodiafiltration. Intensive Care Med 2003;29: 2094-2095.

130 Pea F, Brollo L, Lugano M, Dal Pos L, Furlanut M: Therapeutic drug monitoringguided high teicoplanin dosage regimen required to treat a hypoalbuminemic renal transplant patient undergoing continuous venovenous hemofiltration. Ther Drug Monit 2001;23:587-588.

131 Wolter K, Claus M, Fritschka E: Pharmacokinetics and dosage recommendations of teicoplanin in patients treated by continuous veno-venous haemodialysis (CVVHD). Eur J Clin Pharmacol 1994;46:179-180.

132 Bellmann R, Falkensammer G, Seger C, Weiler S, Kountchev J, Joannidis M: Teicoplanin pharmacokinetics in critically ill patients on continuous veno-venous hemofiltration. Int J Clin Pharmacol Ther 2010;48: 243-249.

133 Zarowitz BJ, Anandan JV, Dumler F, Jayashankar J, Levin N: Continuous arteriovenous hemofiltration of aminoglycoside antibiotics in critically ill patients. J Clin Pharmacol 1986;26:686-689.

134 Ernest D, Cutler DJ: Gentamicin clearance during continuous arteriovenous hemodiafiltration. Crit Care Med 1992;20:586-589.

135 Syka M, Markantonis SL, Mathas C, Maggina N: A pilot study of netilmicin pharmacokinetics during continuous venovenous hemodiafiltration. J Clin Pharmacol 2005, 45:477-481.
136 Robert R, Rochard E, Malin F, Bouquet S: Amikacin pharmacokinetics during continuous veno-venous hemofiltration. Crit Care Med 1991;19:588-589.

137 Moon SY, Oh KH, Oh YK, Ann C, Joo KW, Kim YS, Han JS, Kim S, Lee JS, Kim JR, Yu KS, Jang IJ, Shin SG: Removal of amikacin in patients undergoing continuous venovenous hemodiafiltration. Korean J Nephrol 2006;25:595-601.

138 Ikawa K, Morikawa N, Suyama H, Ikeda K, Yamanoue T: Pharmacokinetics and pharmacodynamics of once-daily arbekacin during continuous venovenous hemodiafiltration in critically ill patients. J Infect Chemother 2009;15:420-423.

139 Li J, Rayner CR, Nation RL, Deans R, Boots $\mathrm{R}$, Widdecombe N, Douglas A, Lipman J: Pharmacokinetics of colistin methanesulfonate and colistin in a critically ill patient receiving continuous venovenous hemodiafiltration. Antimicrob Agents Chemother 2005;49:4814-4815.

140 Fiaccadori E, Maggiore U, Rotelli C, Giacosa R, Parenti E, Picetti E, Sagripanti S, Manini P, Andreoli R, Cabassi A: Removal of linezolid by conventional intermittent hemodialysis, sustained low-efficiency dialysis, or continuous venovenous hemofiltration in patients with acute renal failure. Crit Care Med 2004;32:2437-2442.

141 Pea F, Viale P, Lugano M, Pavan F, Scudeller L, Della Rocca G, Furlanut M: Linezolid disposition after standard dosages in critically ill patients undergoing continuous venovenous hemofiltration: a report of 2 cases. Am J Kidney Dis 2004;44:1097-1102.

- 142 Meyer B, Kornek GV, Nikfardjam M, Karth GD, Heinz G, Locker GJ, Jaeger W, Thalhammer F: Multiple-dose pharmacokinetics of linezolid during continuous venovenous haemofiltration. J Antimicrob Chemother 2005;56:172-179.

143 Churchwell MD, Pasko DA, Mueller BA: Daptomycin clearance during modeled continuous renal replacement therapy. Blood Purif 2006;24:548-554.

144 Schetz M, Ferdinande P, Van den Berghe G, Verwaest C, Lauwers P: Pharmacokinetics of continuous renal replacement therapy. Intensive Care Med 1995;21:612-620. 